\title{
Theoretical Analysis on the Nonlinear Free Vibration of a Tri-Cross String
}

\author{
Bo Pan, ${ }^{1}$ Jingda Tang, ${ }^{2}$ Ryuichi Tarumi, ${ }^{3}$ Fulin Shang, ${ }^{2}$ Yanbo Wang, \\ Weijun Zhang, ${ }^{1}$ and Xueyan Zhang ${ }^{1}$ \\ ${ }^{1} X i$ 'an Thermal Power Research Institute Co., Ltd., Xian, Shaanxi 710032, China \\ ${ }^{2}$ State Key Lab for Strength and Vibration of Mechanical Structures, Department of Engineering Mechanics, \\ Xian Jiaotong University, Xian 710049, China \\ ${ }^{3}$ Department of Mechanical Engineering, Osaka University, Suita, Osaka 565-0871, Japan
}

Correspondence should be addressed to Bo Pan; panbo@tpri.com.cn

Received 9 July 2017; Accepted 16 October 2017; Published 20 November 2017

Academic Editor: Chao Tao

Copyright (C) 2017 Bo Pan et al. This is an open access article distributed under the Creative Commons Attribution License, which permits unrestricted use, distribution, and reproduction in any medium, provided the original work is properly cited.

\begin{abstract}
Here we present a theoretical analysis on the nonlinear free vibration of a tri-cross string system, which is an element of space net-antennas. We derived the governing equations from Hamilton's principle and obtained a linearized solution by the standard perturbation method. The semi-analytical solutions of the governing equations have not been provided referring to the solution of plate vibrating problem. This analysis revealed that natural frequencies of the tri-cross string depend on the vibration amplitude due to the geometrical nonlinearity in the constitutive equation. The geometric parameters, such as the diameters and the lengths of the constituent strings, also affect the frequency through the nonlinearity of the tri-cross string. The nonlinear natural frequency shows coupled characteristic; that is, the natural frequency of the tri-cross string varies with that of the constituent strings, but the contribution of each constituent string to the natural frequency is in different proportions.
\end{abstract}

\section{Introduction}

Recently, we have analyzed the nonlinear dynamic response of the cross string, the simplest net structure of strings such as the space antennas, using Hamilton's principle and the perturbation method [1]. Tri-cross string is another simplest net structure with odd constituent strings among all the multi-cross strings, except for the cross string. It is difficult to discuss their oscillation characteristics (such as natural frequency) by a theoretical analysis because of the complex nonlinearity. However, it is necessary to provide an analytical result to compare with experiments to obtain insight on the motion law of the structure and guide the structural design.

Natural vibration of an initially stressed and linearly elastic string with small amplitudes has been extensively investigated by theoretical analysis, simulations, and experimental researches. However, the relevant researches on the initially stretched string with finite amplitudes are still rare, despite they are quite important for the engineering problems. The Kirchhoff string equation is accepted as a good first approximation of the nonlinear behavior in the transverse direction of a string [2]. Molteno and Tufillaro studied qualitatively the agreement between the analytical results obtained via the truncated Kirchhoff string equation and the experimental results [3]. Various numerical schemes, such as the Galerkin method, were developed to simulate the largeamplitude vibrating problem of the elastic strings based on the nonlinear Kirchhoff string model [4]. Xiong and Hutton used Hamilton's principle to obtain the governing equation and the boundary conditions of a multi-guided rotating string [5], and they proved that Hamilton's principle could be used for the derivation of nonlinear vibration problems as we presented in a recent paper [1]. Later on, more complicated conditions of single string vibrating problem were analyzed, such as moving boundary conditions [6], unsteady vibration varying length [7], and the influence of friction force [8]. The transverse vibration of nonlinear strings is governed by partial differential equations. A finite difference method has been developed to obtain the numerical solutions of 


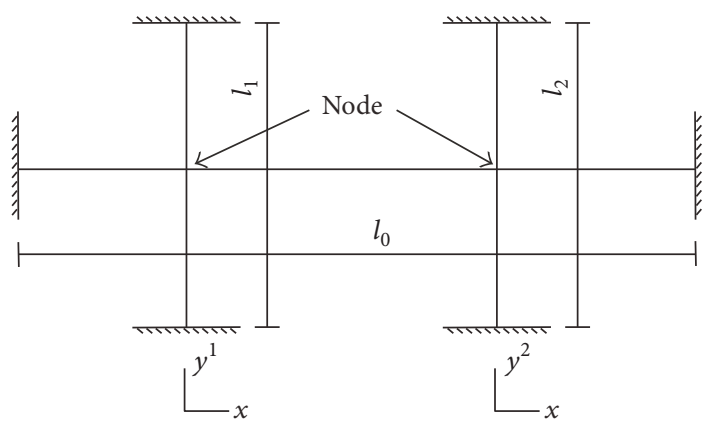

(a)

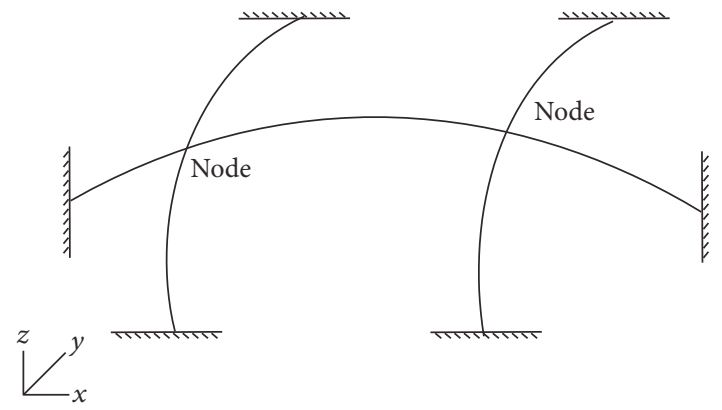

(b)

Figure 1: (a) The initial state of a tri-cross string. (b) The oscillating state of a tri-cross string.

these equations using the perturbation method [9], different from the treatment of Carrier $[10,11]$. The mentioned studies mainly focus on the vibrating characteristics of a single elastic string. The experimental and computational researches of networks and space antennas most focus on the cases of linear vibration $[12,13]$ and linear design $[14,15]$, while the complex nonlinear behavior of the multistring networks is still an open issue. However, few theoretical works on the multistring networks were found in the literature. In this work, we further consider the problem of a tri-cross string, which is another simplest net structure with odd constituent strings and the theoretical studies on tri-cross strings are necessary, especially for the space antennas.

In this study, we studied the free nonlinear vibration response of a tri-cross string, composed of three single strings as shown in Figure 1, and attempted to provide a theoretical method to analyze the nonlinear features of the tri-cross string. The governing equations of the nonlinear vibration of the tri-cross string are derived from Hamilton's principle and solved by the perturbation method. As the traditional potential-function method used for the nonlinear vibration of a single string is quite difficult to adopt for a tri-cross string, novel semi-analytical solutions of the governing equations were provided. Based on the obtained solutions, we discuss the influence of the nonlinear feature and coupling properties on the total frequency of the tri-cross string. The results show that the contribution of each constituent string to the tricross natural frequency is in different proportions. Moreover, the frequencies of the tri-cross string are dependent on the material properties of its constituent strings. The work may provide theoretical guide for the free vibration of net structures such as space antennas with flexible strings.

\section{Derivation of the Motion Equations}

2.1. Model. As shown in Figure 1, the three single strings are connected to each other by two joints (later referred to as node) in the tri-cross string, and these two nodes will keep connected during vibration. This assembled structure known as tri-cross string can be treated as another kind of the simplest net of strings except for the cross string in [1]. In this study, the condition of geometrical nonlinearity and material linearity is considered. It means the motion of the tri-cross string is transverse and one-dimensional with large amplitude, but the elongation of the string during vibration is not negligible. Furthermore, the strains in these strings remain elastic in the linear range. In nonlinear analysis, the tension in the tri-cross should vary and can usually be expressed as the function of position and time, that is, $T(x, t)$. For simplicity, the tension force can be further treated as the only function of time $T(t)[2,11]$. In Figure 1 , the axis is applied to connect to each string. The strings connected to $y^{1}$-axis and $y^{2}$-axis are jointed to the string of $x$-axis, which are called $y^{1}$-axis and $y^{2}$-axis for short, respectively. During the vibration, the vibrated modal of $y^{1}$-axis and $y^{2}$-axis can be different orders and would differ in the characteristics of vibrating plane. However, they are both connected to the $x$ string and affected by each other by the constraint of nodes. Considering the real structure of net strings such as space antennas, the nonrigid boundary conditions and the finite span of this tri-cross string are adopted in this paper. For the sake of simplicity, the three single strings are chosen to be made of the same material as well.

In the following section, we will try to simplify the structure of the tri-cross string and transfer it into a dynamic model. At first, the whole jointed tri-cross string structure can be divided into three single strings with two nodal forces applied onto each of them. Considering jogged two strings of the whole tri-cross string, the separated nodal force on each of the jogged two strings has the same amplitude but in the opposite direction. In this study, the jogged tri-cross string will be disassembled into the three strings separately, and one of them will be chosen as an object substituted into Hamilton's principle. In this way, the governing equation of one single string belonging to the jogged tri-cross string structure can be obtained, and it can demonstrate the common properties of each single string.

Under the above assumptions, the governing equations of the vibration of the tri-cross string are derived as follows.

2.2. Hamilton's Principle. The Lagrange integral function $J$ of a single string can be expressed as

$$
J=\int_{t_{1}}^{t_{2}} L(t) \mathrm{d} t
$$




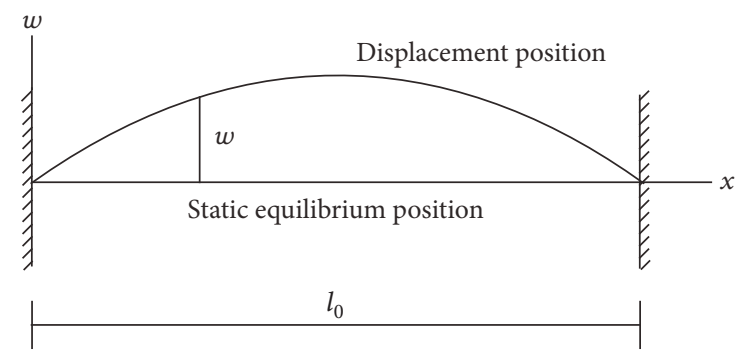

FIGURE 2: Displacement of the $x$-string component of the cross string in Figure 1.

where $J$ is obtained by integrating Lagrange function $L$ with respect to the time $t$ and $L$ is expressed as

$$
L(t)=K(t)-U(t),
$$

where $K$ is the kinetic energy and $U$ is the potential energy of the system.

As the derivation method and process of the mechanics models for the three constituent single strings of the tri-cross string are similar, it is convenient to present the derivation of the mathematical expressions of $K(t)$ and $U(t)$ of one string.

In the present problem, the vibrating direction is vertical to $x-y$ plane. Therefore, the expression of the kinetic energy $K(t)$ is shown as

$$
K(t)=\frac{1}{2} \int_{0}^{l_{0}} \rho\left(\frac{\partial w}{\partial t}\right)^{2} \mathrm{~d} x,
$$

where $l_{0}$ is the length of the string in the initial static equilibrium position, $\rho$ is the mass per unit length and is the function of $x, w$ is the displacement of the string, and $x$ is the longitudinal coordinate.

The total potential energy $U(t)$ of one string consists of one part due to tension work $U_{T}(t)$ and the other part coming from the external excitation $U_{F}(t)$, i.e., $U(t)=U_{T}(t)+U_{F}(t)$. As shown in Figure 2, a string in the equilibrium position presents an initial tension $T_{0}$ and its initial length $l_{0}$. We take an infinitesimal element of the displaced string as an object, and its length in the deformed configuration is $\mathrm{d} s$. In the equilibrium position under initial tension, the length of this element is $\mathrm{d} x$, under the definition of initial length being $\mathrm{d} x_{0}$. It is assumed that the string remains linearly elastic under the varying tension during the vibration, and the tension $T(x, t)$ can be expressed as

$$
T(x, t)=E A \varepsilon,
$$

in which the total strain of the element $\varepsilon$ is

$$
\varepsilon=\frac{\mathrm{d} s-\mathrm{d} x_{0}}{\mathrm{~d} x_{0}}=\frac{\mathrm{d} s}{\mathrm{~d} x} \frac{\mathrm{d} x}{\mathrm{~d} x_{0}}, \quad \frac{\mathrm{d} x}{\mathrm{~d} x_{0}}=1+\varepsilon_{0},
$$

where $\varepsilon_{0}$ is the initial strain. According to the geometrical relations, the vibrating string away from the initial equilibrium position can be expressed as

$$
\frac{\mathrm{d} s}{\mathrm{~d} x}=\sqrt{1+\left(\frac{\partial w}{\partial x}\right)^{2}} .
$$

By inserting (5) and (6) into (4), (4) can be expressed as

$$
T(x, t)=E A\left[\left(1+\varepsilon_{0}\right) \sqrt{1+\left(\frac{\partial \omega}{\partial x}\right)^{2}}-1\right],
$$

where $E$ is Young's modulus and $A$ is the cross-sectional area. For simplicity and emphasis of geometrically nonlinear behavior, the tension in the one-dimensional and flexible string can be regarded as the function of time only $[2,11]$. With the solution of the nonlinear vibration problem of twodimensional thin plates, averaging the stress in one axis is one effective method. In our previous study, the tension is presented under the same assumption [1]

$$
T(t)=T_{0}+\frac{E A}{l_{0}}\left(\int_{0}^{l_{0}} \sqrt{1+\left(\frac{\partial w(x, t)}{\partial x}\right)^{2}} \mathrm{~d} x-l_{0}\right)
$$

or

$T(t)$

$$
=\frac{E A+T_{0}}{l_{0}}\left[\int_{0}^{l_{0}} \sqrt{1+\left(\frac{\partial w(x, t)}{\partial x}\right)^{2}} \mathrm{~d} x-\frac{l_{0}}{1+\varepsilon_{0}}\right] .
$$

Using (9), the tension work stored in the potential energy of the one single string with a length of $\mathrm{d} x$ can be expressed as

$$
\begin{gathered}
\mathrm{d} U_{T}(t)=\frac{E A+T_{0}}{l_{0}}\left[\int_{0}^{l_{0}} \sqrt{1+\left(\frac{\partial w}{\partial x}\right)^{2}} \mathrm{~d} x-\frac{l_{0}}{1+\varepsilon_{0}}\right] \\
\cdot\left[\sqrt{1+\left(\frac{\partial w}{\partial x}\right)^{2}} \mathrm{~d} x-\mathrm{d} x\right] .
\end{gathered}
$$

Integrating (10) along the string, we can obtain

$$
\begin{aligned}
U_{T}(t)= & \frac{E A+T_{0}}{4 l_{0}}\left[\int_{0}^{l_{0}}\left(\frac{\partial w}{\partial x}\right)^{2} \mathrm{~d} x\right] \\
& \cdot\left\{\int_{0}^{l_{0}}\left[\frac{2 \varepsilon_{0}}{1+\varepsilon_{0}}+\left(\frac{\partial w}{\partial x}\right)^{2}\right] \mathrm{d} x\right\} .
\end{aligned}
$$

On the other hand, one external excitation work stored in the potential energy is provided as

$$
\begin{aligned}
U_{F}(t)= & \int_{0}^{l_{0}} F_{\text {joint }}^{1}(t) \cdot \delta\left(x-x_{\text {joint }}\right) \\
& \cdot w_{\text {st }}\left(x, y_{\text {joint }}^{1}, y_{\text {joint }}^{2}, t\right) \mathrm{d} x,
\end{aligned}
$$

where $F_{\text {joint }}^{1}(t), F_{\text {joint }}^{2}(t)$ denote the nodal forces at the joints, whose coordinates are $\left(x_{1, \text { joint }}, y_{\text {joint }}^{1}, y_{\text {joint }}^{2}\right),\left(x_{2, \text { joint }}\right.$, $\left.y_{\text {joint }}^{1}, y_{\text {joint }}^{2}\right)$, respectively; $w_{\text {st }}$ represents the displacement of the whole tri-cross string; $\delta(x)$ is Dirac delta function. Figure 3 shows the relation between the (inner) force and the excitation. In fact, the nodal force should be regarded as an inner constraint force by considering the whole 


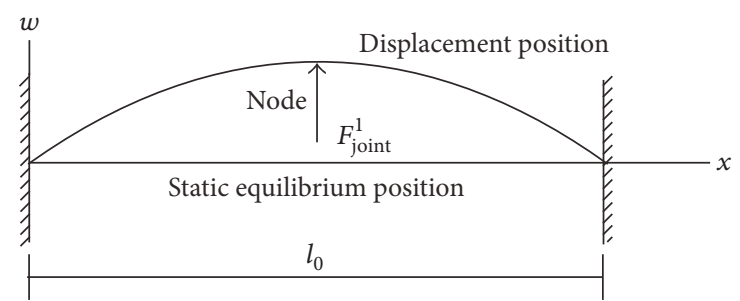

FIGURE 3: Modeling of the tri-cross string by separating the three strings of the structure and adding a joint force to the joint as the joint condition.

tri-cross string. By dividing the tri-cross string into three strings, the nodal forces in the same position but belonging to two different jogged strings have the same amplitude and opposite direction.

For one single string with more than one joint with other constituent strings under multiple excitations, say, along $x$ direction, (12) may be extended as

$$
\begin{aligned}
& U_{F}(t)=\int_{0}^{l_{0}} \sum_{i=1}^{n} F_{\text {joint }}^{i}(t) \cdot \delta\left(x-x_{i, \text { joint }}\right) \\
& \cdot w_{\text {st }}\left(x, y_{\text {joint }}^{1}, y_{\text {joint }}^{2}, t\right) \mathrm{d} x .
\end{aligned}
$$

According to Hamilton's principle, (1) can be expressed by substituting (11), (12), (13) into (1) as

$$
\delta J=\delta \int_{t_{1}}^{t_{2}}\left[K(t)-U_{T}(t)-U_{F}(t)\right] \mathrm{d} t=0 .
$$

2.3. Governing Equations. According to (14) and the differential variational principle, the governing equation of the $x$ string can be obtained as

$$
\begin{aligned}
& \rho \frac{\partial^{2} w}{\partial t^{2}}-\frac{E A+T_{0}}{l_{0}}\left(\left(\int_{0}^{l_{0}}\left(\frac{\partial w}{\partial x}\right)^{2} \mathrm{~d} x\right)+\frac{\varepsilon_{0} l_{0}}{1+\varepsilon_{0}}\right) \\
& \quad \cdot \frac{\partial^{2} w}{\partial x^{2}}=F_{\text {joint }}^{1}(t) \cdot \delta\left(x-x_{1, \text { joint }}\right)+F_{\text {joint }}^{2}(t) \\
& \cdot \delta\left(x-x_{2, \text { joint }}\right), \\
& y^{1}=y_{\text {joint }}^{1}, \\
& y^{2}=y_{\text {joint }}^{2} .
\end{aligned}
$$

Similarly, by applying the kinetic energy $K(t)$ and the potential energy $U(t)$ on $y^{1}$-string and $y^{2}$-string, we can obtain the governing equations of $y^{1}$-string and $y^{2}$-string as

$$
\begin{aligned}
& \rho \frac{\partial^{2} w^{\prime}}{\partial t^{2}}-\frac{E A+T_{1}}{l_{1}}\left(\left(\int_{0}^{l_{1}}\left(\frac{\partial w^{\prime \prime}}{\partial y^{1}}\right)^{2} \mathrm{~d} y\right)+\frac{\varepsilon_{1} l_{1}}{1+\varepsilon_{1}}\right) \\
& \quad \cdot \frac{\partial}{\partial y^{1}} \frac{\partial w^{\prime}}{\partial y^{1}}=-F_{\text {joint }}^{1}(t) \cdot \delta\left(y^{1}-y_{\text {joint }}^{1}\right), \\
& x=x_{1, \text { joint }}, \\
& y^{2}=y_{\text {joint }}^{2},
\end{aligned}
$$

$$
\begin{aligned}
& \rho \frac{\partial^{2} w^{\prime \prime}}{\partial t^{2}}-\frac{E A+T_{2}}{l_{2}}\left(\left(\int_{0}^{l_{2}}\left(\frac{\partial w^{\prime \prime}}{\partial y^{2}}\right)^{2} \mathrm{~d} z\right)+\frac{\varepsilon_{2} l_{2}}{1+\varepsilon_{2}}\right) \\
& \quad \cdot \frac{\partial}{\partial y^{2}} \frac{\partial w^{\prime \prime}}{\partial y^{2}}=-F_{\text {joint }}^{2}(t) \cdot \delta\left(y^{2}-y_{\text {joint }}^{2}\right), \\
& x=x_{2, \text { joint }}, \\
& y^{1}=y_{\text {joint }}^{1}
\end{aligned}
$$

in which $l_{1}, l_{2}$ are the lengths of $y^{1}$-string and $y^{2}$-string in the static equilibrium position, respectively; $w, w^{\prime}, w^{\prime \prime}$ are the displacements of $x$-string, $y^{1}$-string, and $y^{2}$-string, respectively; and $w_{\mathrm{st}}$ is the displacement of the whole tri-cross string structure,

$$
\begin{aligned}
w & =w_{\text {st }}\left(x, y_{\text {joint }}^{1}, y_{\text {joint }}^{2}, t\right), \\
w^{\prime} & =w_{\text {st }}\left(x_{1, \text { joint }}, y^{1}, y_{\text {joint }}^{2}, t\right), \\
w^{\prime \prime} & =w_{\text {st }}\left(x_{2, \text { joint }}, y_{\text {joint }}^{1}, y^{2}, t\right) .
\end{aligned}
$$

The boundary conditions corresponding to $x$-string, $y^{1}$ string, and $y^{2}$-string are shown as follows:

$$
\begin{gathered}
w=0, \quad \text { at } x=0, x=l_{0}, \\
w^{\prime}=0, \quad \text { at } y^{1}=0, y^{1}=l_{1}, \\
w^{\prime \prime}=0, \quad \text { at } y^{2}=0, y^{2}=l_{2},
\end{gathered}
$$

and the initial conditions corresponding to $x$-string, $y^{1}$-string, and $y^{2}$-string are

$$
\left.w_{\text {st }}\left(x, y^{1}, y^{2}\right)\right|_{t=0} ^{x=x_{i}, y^{1}=y_{j}^{1}, y^{2}=y_{k}^{2},}=w_{\text {st }}\left(x_{i}, y_{j}^{1}, y_{k}^{2}, 0\right)
$$

where $w_{\text {st }}\left(x^{i}, y^{j}, z^{k}, 0\right)$ is the initial displacement.

It is well known that the natural frequency with nonlinearity of a geometrically nonlinear string is composed of the linear natural frequency of the string and the nonlinear fluctuation frequency $[16,17]$. Based on the above derivations, it is obvious that the vibration of the tri-cross string is coupled with each other due to the two joint nodes. In the following, we will discuss the nonlinearity and the coupling behavior among the $x$-string, $y^{1}$-string, and $y^{2}$-string with the two jogged mechanical connections.

\section{Solution of the Governing Equations}

In the integrodifferential nonlinear governing equations (15a), (15b), and (15c), the second terms all present the term $\partial w / \partial x$. It is difficult to obtain the analytical solutions of the above integrodifferential nonlinear governing equations. In this study, modal superposition method is adopted here to solve these equations with semi-analytical formula first, and the solutions were used to analyze their responses with coupled behavior.

The boundary and initial conditions of the tri-cross string are one kind of a net or special plate; thus similarly 
the tri-trigonometric series can be employed to expand the displacement of the tri-cross string as

$$
\begin{aligned}
w_{\mathrm{st}}(x, y, z, t)= & \sum_{m=1}^{M} \sum_{n_{1}=1}^{N 1} \sum_{n_{2}=1}^{N 2} a_{m n_{1} n_{2}} f_{m n_{1} n_{2}}(t) \sin \frac{m \pi x}{l_{0}} \\
& \cdot \sin \frac{n_{1} \pi y^{1}}{l_{1}} \sin \frac{n_{2} \pi y^{2}}{l_{2}},
\end{aligned}
$$

in which $a_{m n_{1} n_{2}}$ is the $m n_{1} n_{2}$ th initial amplitude of the vibration and $f_{m n_{1} n_{2}}(t)$ presents the dimensionless timedomain function. In the tri-cross string, the time dependence of $x$-string, $y^{1}$-string, and $y^{2}$-string is exactly the same. Moreover, in order to make (9) satisfy the initial condition, assume $f_{m n_{1} n_{2}}(t=0)=1$. Considering the geometrical structure in Figure 1, the magnitude of $w_{\text {st }}$ is zero when $x \neq x_{1, \text { joint }}$ or $y^{1} \neq y_{\text {joint }}^{1}, y^{1} \neq y_{\text {joint }}^{1}$ or $y^{2} \neq y_{\text {joint }}^{2}$, and $x \neq x_{2, \text { joint }}$ or $y^{2} \neq y_{\text {joint }}^{2}$. Lining to (8) and (19), all $a_{m n_{1} n_{2}} \mathrm{~s}$ should be equal to zero based on the initial and boundary conditions. Therefore, it is obvious that $w_{\mathrm{st}}$ is nonzero only at $\left(x, y_{\text {joint }}^{1}, y_{\text {joint }}^{2}\right)$ or $\left(x_{1, \text { joint }}, y^{1}, y_{\text {joint }}^{2}, t\right)$ or $\left(x_{2, \text { joint }}, y_{\text {joint }}^{1}, y^{2}, t\right)$ according to the geometrical structure shown in Figure 1.

Considering the corresponding external excitation terms in (15a), (15b), and (15c), we can describe the nodal forces in modal form as trigonometric series

$$
\begin{aligned}
F_{\text {joint }}^{1}(t) \cdot \delta\left(x-x_{1, \text { joint }}\right) & =\sum_{m=1}^{M} F_{m}^{1 * *} \sin \frac{m \pi x}{l_{0}}, \\
F_{\text {joint }}^{2}(t) \cdot \delta\left(x-x_{2, \text { joint }}\right) & =\sum_{m=1}^{M} F_{m}^{2 * *} \sin \frac{m \pi x}{l_{0}}, \\
-F_{\text {joint }}^{1}(t) \cdot \delta\left(y^{1}-y_{\text {joint }}^{1}\right) & =\sum_{n_{1}=1}^{N 1} F_{n_{1}}^{1 *} \sin \frac{n_{1} \pi y^{1}}{l_{0}}, \\
-F_{\text {joint }}^{2}(t) \cdot \delta\left(y^{2}-y_{\text {joint }}^{2}\right) & =\sum_{n_{2}=1}^{N 2} F_{n_{2}}^{2 *} \sin \frac{n_{2} \pi y^{2}}{l_{2}},
\end{aligned}
$$

where $F_{m}^{1 * *}, F_{m}^{2 * *}, F_{n_{1}}^{1 *}$, and $F_{n_{2}}^{2 *}$ are the trigonometric modal parameters.

In this paper, we aim to consider the effect of geometrically nonlinear displacement. The length of the string varies rather than being constant. Meanwhile, we suppose the weak nonlinearity of this problem such that the strains inside the tri-cross string are not quite large during vibration. Thus, the amplitudes of displacement $\left(a_{m n_{1} n_{2}}\right)$ are small in magnitude; as a result, the nodal force is also quite small. By substituting (19) and (20a) and (20b) into (15a), we can rewrite the governing equation (15a), for simplicity,

$$
\begin{aligned}
& \rho \ddot{f}_{m n_{1} n_{2}}(t) \\
& +\frac{E A+T_{0}}{l_{0}}\left(\left(\int_{0}^{l_{0}} \sum_{i=1}^{M} \sum_{j=1}^{N 1} \sum_{k=1}^{N 1} \sum_{l=1}^{N 2} \sum_{h=1}^{N 2}\left(\frac{i \pi}{l_{0}}\right)^{2} a_{i j l} a_{i k h} f_{i j l}(t) f_{i k h}(t)\right.\right. \\
& \left.\cdot \cos ^{2} \frac{i \pi x}{l_{0}} \cdot \sin \frac{j \pi y_{\text {joint }}^{1}}{l_{1}} \sin \frac{l \pi y_{\text {joint }}^{2}}{l_{2}} \cdot \sin \frac{k \pi y_{\text {joint }}^{1}}{l_{1}} \sin \frac{h \pi y_{\text {joint }}^{2}}{l_{2}}\right) \\
& \left.+\frac{\varepsilon_{0} l_{0}}{1+\varepsilon_{0}}\right) \cdot\left(\frac{m \pi}{l_{0}}\right)^{2} f_{m n_{1} n_{2}}(t)=\frac{\left(F_{m}^{1 * *}+F_{m}^{2 * *}\right)}{\left(S_{y z}^{1 * *} a_{m n_{1} n_{2}}\right)}
\end{aligned}
$$

or

$$
\begin{aligned}
& \rho \ddot{f}_{m n_{1} n_{2}}(t) \\
& +\frac{E A+T_{0}}{2}\left(\sum_{i=1}^{M} \sum_{j=1}^{N 1} \sum_{k=1}^{N 1} \sum_{l=1}^{N 2} \sum_{h=1}^{N 2}\left(\frac{i \pi}{l_{0}}\right)^{2} a_{i j l} a_{i k h} f_{i j l}(t) f_{i k h}(t)\right. \\
& \left.\cdot \sin \frac{j \pi y_{\text {joint }}^{1}}{l_{1}} \sin \frac{l \pi y_{\text {joint }}^{2}}{l_{2}} \cdot \sin \frac{k \pi y_{\text {joint }}^{1}}{l_{1}} \sin \frac{h \pi y_{\text {joint }}^{2}}{l_{2}}\right) \\
& \cdot f_{m n_{1} n_{2}}(t)\left(\frac{m \pi}{l_{0}}\right)^{2}+\frac{E A+T_{0}}{l_{0}} \frac{\varepsilon_{0} l_{0}}{1+\varepsilon_{0}} \cdot\left(\frac{m \pi}{l_{0}}\right)^{2} f_{m n_{1} n_{2}}(t) \\
& =\frac{\left(F_{m}^{1 * *}+F_{m}^{2 * *}\right)}{\left(S_{y z}^{1 * *} a_{m n_{1} n_{2}}\right)},
\end{aligned}
$$

where

$$
\begin{aligned}
S_{y z}^{1 * *} & =\sum_{n_{1}=1}^{N 1} \sum_{n_{2}=1}^{N 2} \sin \frac{n_{1} \pi y_{\text {joint }}^{1}}{l_{1}} \sin \frac{n_{2} \pi y_{\text {joint }}^{2}}{l_{2}}, \\
S_{x z}^{1 *} & =\sum_{m=1}^{M} \sum_{n_{1}=1}^{N 2} \sin \frac{m \pi x_{1, \text { oint }}}{l_{0}} \sin \frac{n_{2} \pi y_{\text {joint }}^{2}}{l_{2}}, \\
S_{y z}^{2 * *} & =\sum_{n_{1}=1}^{N 1} \sum_{n_{2}=1}^{N 2} \sin \frac{n_{1} \pi y_{\text {joint }}^{1}}{l_{1}} \sin \frac{n_{2} \pi y_{\text {joint }}^{2}}{l_{2}}, \\
S_{x y}^{2 *} & =\sum_{m=1}^{M} \sum_{n_{1}=1}^{N 1} \sin \frac{m \pi x_{2, \text { joint }}}{l_{0}} \sin \frac{n_{1} \pi y_{\text {joint }}^{1}}{l_{1}} .
\end{aligned}
$$

The orthogonality of trigonometric functions is applied in the derivation of (21a) and (21b). By inserting (19) and (20b) into (15b), we can get similar governing equation for $y^{1}$-string as

$$
\begin{aligned}
& \rho \ddot{f}_{m n_{1} n_{2}}(t)+\frac{E A_{1}+T_{1}}{2}\left(\sum_{i=1}^{M} \sum_{k=1}^{M} \sum_{j=1}^{N 1} \sum_{l=1}^{N 2} \sum_{h=1}^{N 2}\left(\frac{j \pi}{l_{1}}\right)^{2}\right. \\
& \cdot a_{i j l} a_{k j h} f_{i j l}(t) f_{k j h}(t) \cdot \sin \frac{i \pi x_{1, \text { joint }}}{l_{0}} \sin \frac{l \pi y_{\text {joint }}^{2}}{l_{2}} \\
& \left.\cdot \frac{k \pi x_{1, \text { joint }}}{l_{0}} \sin \frac{h \pi y_{\text {joint }}^{2}}{l_{2}}\right) \cdot f_{m n_{1} n_{2}}(t)\left(\frac{n_{1} \pi}{l_{1}}\right)^{2} \\
& +\frac{E A_{1}+T_{1}}{l_{1}} \frac{\varepsilon_{1} l_{1}}{1+\varepsilon_{1}} \cdot\left(\frac{n_{1} \pi}{l_{1}}\right)^{2} f_{m n_{1} n_{2}}(t) \\
& =\frac{F_{n_{1}}^{1 *}}{\left(S_{x z}^{1 *} a_{m n_{1} n_{2}}\right)} .
\end{aligned}
$$

Similarly, substituting (19) and (20c) into (15c), we have similar form of the governing equation for $y^{2}$-string as

$$
\begin{gathered}
\rho \ddot{f}_{m n_{1} n_{2}}(t)+\frac{E A_{2}+T_{2}}{2}\left(\sum_{i=1}^{M} \sum_{k=1}^{M} \sum_{j=1}^{N 1} \sum_{l=1}^{N 1} \sum_{h=1}^{N 2}\left(\frac{h \pi}{l_{2}}\right)^{2}\right. \\
\cdot a_{i j h} a_{k l h} f_{i j h}(t) f_{k l h}(t) \cdot \sin \frac{i \pi x_{2, \text { joint }}}{l_{0}} \sin \frac{j \pi y_{\text {joint }}^{1}}{l_{1}}
\end{gathered}
$$




$$
\begin{aligned}
& \left.\cdot \frac{k \pi x_{2, \text { joint }}}{l_{0}} \sin \frac{l \pi y_{\text {joint }}^{1}}{l_{1}}\right) \cdot f_{m n_{1} n_{2}}(t)\left(\frac{n_{2} \pi}{l_{2}}\right)^{2} \\
& +\frac{E A_{2}+T_{2}}{l_{2}} \frac{\varepsilon_{2} l_{2}}{1+\varepsilon_{2}} \cdot\left(\frac{n_{2} \pi}{l_{2}}\right)^{2} f_{m n_{1} n_{2}}(t) \\
& =\frac{F_{n_{2}}^{2 *}}{\left(S_{x y}^{2 *} a_{m n_{1} n_{2}}\right)} .
\end{aligned}
$$

In order to connect (21a), (23a), and (23b), (20a), (20b), (20c), and (20d) should be rewritten as follows:

$$
\begin{aligned}
& \int_{0}^{l_{0}} F_{\text {joint }}^{1}(t) \cdot \delta\left(x-x_{1, \text { joint }}\right) \sin \frac{m \pi x}{l_{0}} \mathrm{~d} x \\
& =\int_{0}^{l_{0}}\left(\sum_{m=1}^{M} F_{m}^{1 * *} \sin \frac{m \pi x}{l_{0}}\right) \sin \frac{m \pi x}{l_{0}} \mathrm{~d} x, \\
& \int_{0}^{l_{0}} F_{\text {joint }}^{2}(t) \cdot \delta\left(x-x_{2, \text { joint }}\right) \sin \frac{m \pi x}{l_{0}} \mathrm{~d} x \\
& =\int_{0}^{l_{0}}\left(\sum_{m=1}^{M} F_{m}^{2 * *} \sin \frac{m \pi x}{l_{0}}\right) \sin \frac{m \pi x}{l_{0}} \mathrm{~d} x, \\
& \int_{0}^{l_{1}}-F_{\text {joint }}^{1}(t) \cdot \delta\left(y-y_{\text {joint }}^{1}\right) \sin \frac{n_{1} \pi y^{1}}{l_{1}} \mathrm{~d} y^{1} \\
& =\int_{0}^{l_{1}}\left(\sum_{n_{1}=1}^{N 1} F_{n_{1}}^{1 *} \sin \frac{n_{1} \pi y^{1}}{l_{1}}\right) \sin \frac{n_{1} \pi y^{1}}{l_{1}} \mathrm{~d} y^{1}, \\
& \int_{0}^{l_{2}}-F_{\text {joint }}^{2}(t) \cdot \delta\left(y^{2}-y_{\text {joint }}^{2}\right) \sin \frac{n_{2} \pi y^{2}}{l_{2}} \mathrm{~d} y^{2} \\
& =\int_{0}^{l_{2}}\left(\sum_{n_{2}=1}^{N 2} F_{n_{2}}^{2 *} \sin \frac{n_{2} \pi y^{2}}{l_{2}}\right) \sin \frac{n_{2} \pi y^{2}}{l_{2}} \mathrm{~d} y^{2} .
\end{aligned}
$$

Based on (24a)-(24d), one has

$$
\begin{aligned}
& F_{m}^{1 * *}=-B_{m n_{1}}^{1} F_{n_{1}}^{1 *}, \\
& F_{m}^{2 * *}=-B_{m n_{2}}^{2} F_{n_{2}}^{2 *},
\end{aligned}
$$

where

$$
B_{m n_{1}}^{1}=\frac{l_{1} \sin \left(m \pi x_{1, \text { joint }} / l_{0}\right)}{l_{0} \sin \left(n_{1} \pi y_{\text {joint }}^{1} / l_{1}\right)}=\frac{l_{1}}{l_{0}} \frac{x_{1}^{m}}{y_{1}^{n_{1}}},
$$

$$
\begin{aligned}
B_{m n_{2}}^{2} & =\frac{l_{2} \sin \left(m \pi x_{2, \text { joint }} / l_{0}\right)}{l_{0} \sin \left(n_{2} \pi y_{\text {joint }}^{2} / l_{2}\right)}=\frac{l_{2}}{l_{1}} \frac{x_{2}^{m}}{y_{2}^{n_{2}}} \\
x_{1}^{m} & =\sin \frac{m \pi x_{1, \text { joint }}}{l_{0}} \\
x_{2}^{m} & =\sin \frac{m \pi x_{2, \text { joint }}}{l_{0}} \\
y_{1}^{n_{1}} & =\sin \frac{n_{1} \pi y_{\text {joint }}^{1}}{l_{1}} \\
y_{2}^{n_{2}} & =\sin \frac{n_{2} \pi y_{\text {joint }}^{2}}{l_{2}}
\end{aligned}
$$

In the perturbation method, a properly chosen small perturbation parameter should be provided during the solution. For achieving this purpose, we defined

$$
\begin{aligned}
g_{m n_{1} n_{2}}(t)= & \left(E A+T_{0}\right)\left(E A_{1}+T_{1}\right)\left(E A_{2}+T_{2}\right)\left(\frac{m \pi}{l_{0}}\right) \\
& \cdot\left(\frac{n_{1} \pi}{l_{1}}\right)\left(\frac{n_{2} \pi}{l_{2}}\right) \frac{l_{0}}{T_{0}} \frac{l_{1}}{T_{1}} \frac{l_{2}}{T_{2}} f_{m n_{1} n_{2}}(t),
\end{aligned}
$$

where

$$
\begin{aligned}
D_{m n_{1} n_{2}}= & \left(E A+T_{0}\right)\left(E A_{1}+T_{1}\right)\left(E A_{2}+T_{2}\right)\left(\frac{m \pi}{l_{0}}\right) \\
& \cdot\left(\frac{n_{1} \pi}{l_{1}}\right)\left(\frac{n_{2} \pi}{l_{2}}\right) \frac{l_{0}}{T_{0}} \frac{l_{1}}{T_{1}} \frac{l_{2}}{T_{2}} .
\end{aligned}
$$

From $(27), f_{m n_{1} n_{2}}(t)$ can be written as

$$
\begin{aligned}
f_{m n_{1} n_{2}}(t)= & \left(\frac{m \pi}{l_{0}}\right)^{-1}\left(\frac{n_{1} \pi}{l_{1}}\right)^{-1}\left(\frac{n_{2} \pi}{l_{2}}\right)^{-1} \\
& \cdot \frac{T_{0}}{\left(E A+T_{0}\right) l_{0}} \frac{T_{1}}{\left(E A_{1}+T_{1}\right) l_{1}} \\
& \cdot \frac{T_{2}}{\left(E A_{2}+T_{2}\right) l_{2}} g_{m n_{1} n_{2}}(t) .
\end{aligned}
$$

By substituting (29) into (22a) and (22b), the governing equation of $x$-string can be transformed from (21a) and (21b) to

$$
\begin{aligned}
& \ddot{g}_{m n_{1} n_{2}}(t)+\frac{T_{0}{ }^{2} T_{1}{ }^{2} T_{2}{ }^{2} l_{0}{ }^{-4} a_{m n_{1} n_{2}}^{2} m^{2}}{4 \rho \pi^{2}\left(E A+T_{0}\right)\left(E A_{1}+T_{1}\right)^{2}\left(E A_{2}+T_{2}\right)^{2}}\left(\sum_{i=1}^{M} \sum_{j=1}^{N 1} \sum_{k=1}^{N 1} \sum_{l=1}^{N 2} \sum_{h=1}^{N 2} \frac{a_{i j l} a_{i k h}}{j k l h a_{m n_{1} n_{2}}^{2}} y_{1}^{j} y_{1}^{k} y_{2}^{l} y_{2}^{h} g_{i j l}(t) g_{i k h}(t)\right) \cdot g_{m n_{1} n_{2}}(t) \\
& +\left(\omega_{0 m}\right)^{2} g_{m n_{1} n_{2}}(t)=\frac{D_{m n_{1} n_{2}}\left(F_{m}^{1 * *}+F_{m}^{2 * *}\right)}{\left(\rho S_{y z}^{1 * *} a_{m n_{1} n_{2}}\right)} .
\end{aligned}
$$


Similarly, the governing equation of $y^{1}$-string becomes

$$
\begin{aligned}
& \ddot{g}_{m n_{1} n_{2}}(t) \\
& +\frac{T_{0}^{2} T_{1}{ }^{2} T_{2}{ }^{2} l_{1}{ }^{-4} a_{m n_{1} n_{2}}^{2} n_{1}^{2}}{4 \rho \pi^{2}\left(E A+T_{0}\right)^{2}\left(E A_{1}+T_{1}\right)\left(E A_{2}+T_{2}\right)^{2}} \\
& \quad \cdot \sum_{i=1}^{M} \sum_{k=1}^{M} \sum_{j=1}^{N 1} \sum_{l=1}^{N 2} \sum_{h=1}^{N 2} \frac{a_{i j l} a_{k j h}}{i k l h a_{m n_{1} n_{2}}^{2}} x_{1}^{i} x_{1}^{k} y_{2}^{l} y_{2}^{h} g_{i j l}(t) g_{k j h}(t) \\
& \cdot g_{m n_{1} n_{2}}(t)+\left(\omega_{0 n_{1}}^{\prime}\right)^{2} g_{m n_{1} n_{2}}(t)=\frac{D_{m n_{1} n_{2}} F_{n_{1}}^{1 *}}{\left(\rho S_{x z}^{1 *} a_{m n_{1} n_{2}}\right)}
\end{aligned}
$$

and the governing equation of $y^{2}$-string becomes

$$
\begin{aligned}
& \ddot{g}_{m n_{1} n_{2}}(t) \\
& +\frac{T_{0}^{2} T_{1}^{2} T_{2}^{2} l_{2}^{-4} a_{m n_{1} n_{2}}^{2} n_{2}^{2}}{4 \rho \pi^{2}\left(E A+T_{0}\right)^{2}\left(E A_{1}+T_{1}\right)^{2}\left(E A_{2}+T_{2}\right)} \\
& \cdot \sum_{i=1}^{M} \sum_{k=1}^{M} \sum_{j=1}^{N 1} \sum_{l=1}^{N 1} \sum_{h=1}^{N 2} \frac{a_{i j h} a_{k l h}}{i j k l a_{m n_{1} n_{2}}^{2}} x_{2}^{i} x_{2}^{k} y_{1}^{l} y_{1}^{h} g_{i j h}(t) g_{k l h}(t) \\
& \cdot g_{m n_{1} n_{2}}(t)+\left(\omega_{0 n_{2}}^{\prime \prime}\right)^{2} g_{m n_{1} n_{2}}(t)=\frac{D_{m n_{1} n_{2}} F_{n_{2}}^{2 *}}{\left(\rho S_{x y}^{2 *} a_{m n_{1} n_{2}}\right)} .
\end{aligned}
$$

As the three single strings have the identical displacements at the joint, the joint condition can be defined as follows:

$$
\begin{aligned}
w\left(x_{1, \text { joint }}, t\right) & =w^{\prime}\left(y_{\text {joint }}^{1}, t\right) \\
& =w_{\text {st }}\left(x_{1, \text { joint }}, y_{\text {joint }}^{1}, y_{\text {joint }}^{2}, t\right), \\
w\left(x_{2, \text { joint }}, t\right) & =w^{\prime \prime}\left(y_{\text {joint }}^{2}, t\right) \\
& =w_{\text {st }}\left(x_{2, \text { joint }}, y_{\text {joint }}^{1}, y_{\text {joint }}^{2}, t\right) .
\end{aligned}
$$

Define

$$
\omega_{0 m}^{2}=\frac{T_{0}}{\rho}\left(\frac{m \pi}{l_{0}}\right)^{2},
$$

where $\omega_{0 m}$ is the linear natural frequency of $x$-string, proportional to the initial tension $T_{0}$ and the square of the string length $l_{0}$. Similarly, the linear natural frequency of $y^{1}$-string and $y^{2}$-string can be defined as

$$
\begin{aligned}
& \left(\omega_{0 n_{1}}^{\prime}\right)^{2}=\frac{T_{1}}{\rho} \cdot\left(\frac{n_{1} \pi}{l_{1}}\right)^{2}, \\
& \left(\omega_{0 n_{2}}^{\prime \prime}\right)^{2}=\frac{T_{2}}{\rho} \cdot\left(\frac{n_{2} \pi}{l_{2}}\right)^{2} .
\end{aligned}
$$

Three small perturbation parameters can be defined as

$$
\begin{aligned}
& \varepsilon_{m n_{1} n_{2}}^{x}=\frac{T_{0}^{2} T_{1}^{2} T_{2}^{2} l_{0}^{-4} a_{m n_{1} n_{2}}^{2} m^{2}}{4 \rho \pi^{2}\left(E A+T_{0}\right)\left(E A_{1}+T_{1}\right)^{2}\left(E A_{2}+T_{2}\right)^{2}}, \\
& \varepsilon_{m n_{1} n_{2}}^{y^{1}}=\frac{T_{0}{ }^{2} T_{1}{ }^{2} T_{2}{ }^{2} l_{1}{ }^{-4} a_{m n_{1} n_{2}}^{2} n_{1}^{2}}{4 \rho \pi^{2}\left(E A+T_{0}\right)^{2}\left(E A_{1}+T_{1}\right)\left(E A_{2}+T_{2}\right)^{2}}, \\
& \varepsilon_{m n_{1} n_{2}}^{y^{2}}=\frac{T_{0}{ }^{2} T_{1}{ }^{2} T_{2}{ }^{2} l_{2}{ }^{-4} a_{m n_{1} n_{2}}^{2} n_{2}^{2}}{4 \rho \pi^{2}\left(E A+T_{0}\right)^{2}\left(E A_{1}+T_{1}\right)^{2}\left(E A_{2}+T_{2}\right)}, \\
& \varepsilon_{m n_{1} n_{2}}^{x}=c_{m n_{1}}^{x-y^{1}} \varepsilon_{m n_{1} n_{2}}^{y^{1}}, \quad c_{m n_{1}}^{x-y^{1}}=\frac{l_{0}^{-4}}{l_{1}^{-4}} \frac{m^{2}}{n_{1}^{2}} \frac{\left(E A+T_{0}\right)}{\left(E A_{1}+T_{1}\right)}, \\
& \varepsilon_{m n_{1} n_{2}}^{x}=c_{m n_{2}}^{x-y^{2}} \varepsilon_{m n_{1} n_{2}}^{y^{2}}, \quad c_{m n_{2}}^{x-y^{2}}=\frac{l_{0}^{-4}}{l_{2}^{-4}} \frac{m^{2}}{n_{2}^{2}} \frac{\left(E A+T_{0}\right)}{\left(E A_{2}+T_{2}\right)} .
\end{aligned}
$$

By substituting (24a), (24b), (24c), (24d), (27), (36), (37), (38), and (39) into (30), (31), and (32), we obtain

$$
\begin{aligned}
& \ddot{g}_{m n_{1} n_{2}}(t)+\frac{\varepsilon_{m n_{1} n_{2}}^{x}}{1+b_{m n_{1}}^{y^{1}}+b_{m n_{2}}^{y^{2}}}\left(\left(\sum_{i=1}^{M} \sum_{j=1}^{N 1} \sum_{k=1}^{N 1} \sum_{l=1}^{N 2} \sum_{h=1}^{N 2} p_{j k l h}\right.\right. \\
& \cdot g_{i j l}(t) g_{i k h}(t) g_{m n_{1} n_{2}}(t)+\frac{b_{m n_{1}}^{y^{1}}}{c_{m n_{1}}^{x-y^{1}}} \\
& \left.\cdot \sum_{i=1}^{M} \sum_{k=1}^{M} \sum_{j=1}^{N 1} \sum_{l=1}^{N 2} \sum_{h=1}^{N 2} q_{i k l h} g_{i j l}(t) g_{k j h}(t) \cdot g_{m n_{1} n_{2}}(t)\right) \\
& +\frac{b_{m n_{2}}^{y^{2}} \sum^{M} \sum_{k=1}^{M} \sum_{j=1}^{N 1} \sum_{l=1}^{N 1} \sum_{h=1}^{N 2} V_{i j k l} g_{i j h}(t) g_{k l h}(t)}{c_{m n_{2}}^{y_{2}}}(t=1) \\
& \left.\cdot g_{m n_{1} n_{2}}(t)\right) \\
& +\frac{\left(\omega_{0 m}\right)^{2}+b_{m n_{1}}^{y^{1}}\left(\omega_{0 n_{1}}^{\prime}\right)^{2}+b_{m n_{2}}^{y^{2}}\left(\omega_{0 n_{2}}^{\prime \prime}\right)^{2}}{1+b_{m n_{1}}^{y^{1}}+b_{m n_{2}}^{y^{2}}} g_{m n_{1} n_{2}}(t) \\
& +0,
\end{aligned}
$$

in which

$$
\begin{aligned}
p_{j k l h} & =\frac{a_{i j l} a_{i k h}}{j k l h a_{m n_{1} n_{2}}^{2}} y_{1}^{j} y_{1}^{k} y_{2}^{l} y_{2}^{h}, \\
q_{i k l h} & =\frac{a_{i j l} a_{k j h}}{i k l h a_{m n_{1} n_{2}}^{2}} x_{1}^{i} x_{1}^{k} y_{2}^{l} y_{2}^{h}, \\
V_{i j k l} & =\frac{a_{i j h} a_{k l h}}{i j k l a_{m n_{1} n_{2}}^{2}} x_{2}^{i} x_{2}^{k} y_{1}^{l} y_{1}^{h},
\end{aligned}
$$




$$
\begin{aligned}
& b_{m n_{1}}^{y^{1}}=\frac{S_{x z}^{1 *} B_{m n_{1}}^{1}}{S_{y z}^{1 * *}}, \\
& b_{m n_{2}}^{y^{2}}=\frac{S_{x y}^{2 *} B_{m n_{2}}^{2}}{S_{y z}^{2 * *}} .
\end{aligned}
$$

By adopting the perturbation method, $g_{m n_{1} n_{2}}(t)$ can be decomposed by means of the perturbation expansion

$$
\begin{aligned}
g_{m n_{1} n_{2}}(t)= & g_{0 m n_{1} n_{2}}(t)+\frac{\varepsilon_{m n_{1} n_{2}}^{x}}{1+b_{m n_{1}}^{y^{1}}+b_{m n_{2}}^{y^{2}}} g_{1 m n_{1} n_{2}}(t) \\
& +\cdots .
\end{aligned}
$$

The frequency of the tri-cross string is also expressed as the following expressions with a small perturbation parameter

$$
\begin{aligned}
\left(\omega_{m n_{1} n_{2}}\right)^{2}= & \frac{\left(\omega_{0 m}\right)^{2}+b_{m n_{1}}^{y^{1}}\left(\omega_{0 n_{1}}^{\prime}\right)^{2}+b_{m n_{2}}^{y^{2}}\left(\omega_{0 n_{2}}^{\prime \prime}\right)^{2}}{1+b_{m n_{1}}^{y^{1}}+b_{m n_{2}}^{y^{2}}} \\
& +\frac{\varepsilon_{m n_{1} n_{2}}^{x}}{1+b_{m n_{1}}^{y^{1}}+b_{m n_{2}}^{y^{2}}} \alpha_{1} \\
& +\left(\frac{\varepsilon_{m n_{1} n_{2}}^{x}}{1+b_{m n_{1}}^{y^{1}}+b_{m n_{2}}^{y^{2}}}\right)^{2} \alpha_{2}+\cdots
\end{aligned}
$$

By inserting (45) and (46) into (42), we obtain the following relation accompanied with the perturbation parameter $\left(\varepsilon_{m n_{1} n_{2}}^{x} /\left(1+b_{n_{1} m}^{y}+b_{n_{1} n_{2}}^{z}\right)\right)^{0}$

$$
\ddot{g}_{0 m n_{1} n_{2}}(t)+\left(\omega_{m n_{1} n_{2}}\right)^{2} g_{0 m n_{1} n_{2}}(t)=0 \text {. }
$$

By satisfying $f_{m n_{1} n_{2}}(0)=1, g_{0 m n_{1} n_{2}}(t)$ can be taken as

$$
g_{0 m n_{1} n_{2}}(t)=D_{m n_{1} n_{2}} \cos \omega_{m n_{1} n_{2}} t .
$$

The terms accompanied with the perturbation parameter $\left(\varepsilon_{m n_{1} n_{2}}^{x} /\left(1+b_{m n_{1}}^{y^{1}}+b_{m n_{2}}^{y^{2}}\right)\right)^{1}$ are

$$
\begin{aligned}
& \ddot{g}_{1 m n_{1} n_{2}}(t)+\left(\omega_{m n_{1} n_{2}}\right)^{2} g_{1 m n_{1} n_{2}}(t)=\alpha_{1} g_{0 m n_{1} n_{2}}(t) \\
& -\left(\sum_{i=1}^{M} \sum_{j=1}^{N 1} \sum_{k=1}^{N 1} \sum_{l=1}^{N 2} \sum_{h=1}^{N 2} p_{j k l h} g_{0 i j l}(t) g_{0 i k h}(t) g_{0 m n_{1} n_{2}}(t)\right. \\
& +\frac{b_{m n_{1}}^{y^{1}}}{c_{m n_{1}}^{x-y^{1}}} \sum_{i=1}^{M} \sum_{k=1}^{M} \sum_{j=1}^{N 1} \sum_{l=1}^{N 2} \sum_{h=1}^{N 2} q_{i k l h} g_{0 i j l}(t) g_{0 k j h}(t) \\
& \left.\cdot g_{0 m n_{1} n_{2}}(t)\right)+\frac{b_{m n_{2}}^{y^{2}}}{c_{m n_{2}}^{x-y^{2}}}
\end{aligned}
$$$$
\cdot \sum_{i=1}^{M} \sum_{k=1}^{M} \sum_{j=1}^{N 1} \sum_{l=1}^{N 1} \sum_{h=1}^{N 2} V_{i j k l} g_{0 i j h}(t) g_{0 k l h}(t) \cdot g_{0 m n_{1} n_{2}}(t) \text {. }
$$

From (49), $g_{1 m n_{1} n_{2}}(t)$ can be obtained as

$$
\begin{aligned}
& g_{1 m n_{1} n_{2}}(t)=\frac{D_{m n_{1} n_{2}}}{4} \sum_{i=1}^{M} \sum_{j=1}^{N 1} \sum_{k=1}^{N 1} \sum_{l=1}^{N 2} \sum_{h=1}^{N 2} p_{j k l h} \\
& \cdot D_{i j l} D_{i k h}\left\{\frac{\cos \left(\omega_{i j l} t+\omega_{i k h} t+\omega_{m n_{1} n_{2}} t\right)-\cos \omega_{m n_{1} n_{2}} t}{\left(\omega_{i j l}+\omega_{i k h}+\omega_{m n_{1} n_{2}}\right)^{2}-\left(\omega_{m n_{1} n_{2}}\right)^{2}}\right. \\
& +\frac{\cos \left(\omega_{i j l} t+\omega_{i k h} t-\omega_{m n_{1} n_{2}} t\right)-\cos \omega_{m n_{1} n_{2}} t}{\left(\omega_{i j l}+\omega_{i k h}-\omega_{m n_{1} n_{2}}\right)^{2}-\left(\omega_{m n_{1} n_{2}}\right)^{2}} \\
& +\frac{\cos \left(\omega_{i j l} t-\omega_{i k h} t+\omega_{m n_{1} n_{2}} t\right)-\cos \omega_{m n_{1} n_{2}} t}{\left(\omega_{i j l}-\omega_{i k h}+\omega_{m n_{1} n_{2}}\right)^{2}-\left(\omega_{m n_{1} n_{2}}\right)^{2}} \\
& \left.+\frac{\cos \left(\omega_{i j l} t-\omega_{i k h} t-\omega_{m n_{1} n_{2}} t\right)-\cos \omega_{m n_{1} n_{2}} t}{\left(\omega_{i j l}-\omega_{i k h}+\omega_{m n_{1} n_{2}}\right)^{2}-\left(\omega_{m n_{1} n_{2}}\right)^{2}}\right\} \\
& +\frac{D_{m n_{1} n_{2}}}{4} \frac{b_{m n_{1}}^{y^{1}}}{c_{m n_{1}}^{x-y^{1}}} \sum_{i=1}^{M} \sum_{k=1}^{M} \sum_{j=1}^{N 1} \sum_{l=1}^{N 2} \sum_{h=1}^{N 2} q_{i k l h} D_{i j l} D_{k j h} \\
& \left\{\frac{\cos \left(\omega_{i j l} t+\omega_{k j h} t+\omega_{m n_{1} n_{2}} t\right)-\cos \omega_{m n_{1} n_{2}} t}{\left(\omega_{i j l}+\omega_{k j h}+\omega_{m n_{1} n_{2}}\right)^{2}-\left(\omega_{m n_{1} n_{2}}\right)^{2}}\right. \\
& +\frac{\cos \left(\omega_{i j l} t+\omega_{k j h} t-\omega_{m n_{1} n_{2}} t\right)-\cos \omega_{m n_{1} n_{2}} t}{\left(\omega_{i j l}+\omega_{k j h}-\omega_{m n_{1} n_{2}}\right)^{2}-\left(\omega_{m n_{1} n_{2}}\right)^{2}} \\
& +\frac{\cos \left(\omega_{i j l} t-\omega_{k j h} t+\omega_{m n_{1} n_{2}} t\right)-\cos \omega_{m n_{1} n_{2}} t}{\left(\omega_{i j l}+\omega_{k j h}-\omega_{m n_{1} n_{2}}\right)^{2}-\left(\omega_{m n_{1} n_{2}}\right)^{2}} \\
& \left.+\frac{\cos \left(\omega_{i j l} t-\omega_{k j h} t-\omega_{m n_{1} n_{2}} t\right)-\cos \omega_{m n_{1} n_{2}} t}{\left(\omega_{i j l}+\omega_{k j h}-\omega_{m n_{1} n_{2}}\right)^{2}-\left(\omega_{m n_{1} n_{2}}\right)^{2}}\right\} \\
& +\frac{D_{m n_{1} n_{2}}}{4} \frac{b_{m n_{2}}^{y^{2}}}{c_{m n_{2}}^{x-y^{2}}} \sum_{i=1}^{M} \sum_{k=1}^{M} \sum_{j=1}^{N 1} \sum_{l=1}^{N 1} \sum_{h=1}^{N 2} V_{i j k l} D_{i j h} D_{k l h} \\
& \cdot\left\{\frac{\cos \left(\omega_{i j h} t+\omega_{k l h} t+\omega_{m n_{1} n_{2}} t\right)-\cos \omega_{m n_{1} n_{2}} t}{\left(\omega_{i j h}+\omega_{k l h}+\omega_{m n_{1} n_{2}}\right)^{2}-\left(\omega_{m n_{1} n_{2}}\right)^{2}}\right. \\
& +\frac{\cos \left(\omega_{i j h} t+\omega_{k j h} t-\omega_{m n_{1} n_{2}} t\right)-\cos \omega_{m n_{1} n_{2}} t}{\left(\omega_{i j l}+\omega_{k j h}+\omega_{m n_{1} n_{2}}\right)^{2}-\left(\omega_{m n_{1} n_{2}}\right)^{2}} \\
& +\frac{\cos \left(\omega_{i j h} t-\omega_{k j h} t+\omega_{m n_{1} n_{2}} t\right)-\cos \omega_{m n_{1} n_{2}} t}{\left(\omega_{i j l}+\omega_{k j h}+\omega_{m n_{1} n_{2}}\right)^{2}-\left(\omega_{m n_{1} n_{2}}\right)^{2}} \\
& \left.+\frac{\cos \left(\omega_{i j h} t-\omega_{k l h} t-\omega_{m n_{1} n_{2}} t\right)-\cos \omega_{m n_{1} n_{2}} t}{\left(\omega_{i j h}+\omega_{k l h}+\omega_{m n_{1} n_{2}}\right)^{2}-\left(\omega_{m n_{1} n_{2}}\right)^{2}}\right\}
\end{aligned}
$$




$$
\begin{aligned}
\alpha_{1}= & \frac{1}{4} \sum_{i=1}^{M} \sum_{j=1}^{N 1} \sum_{k=1}^{N 1} \sum_{l=1}^{N 2} \sum_{h=1}^{N 2} p_{j k l h} D_{i j l} D_{i k h}+\frac{1}{4} \frac{b_{m n_{1}}^{y^{1}}}{c_{m n_{1}}^{x-y^{1}}} \\
& \cdot \sum_{i=1}^{M} \sum_{k=1}^{M} \sum_{j=1}^{N 1} \sum_{l=1}^{N 2} \sum_{h=1}^{N 2} q_{i k l h} D_{i j l} D_{k j h}+\frac{1}{4} \frac{b_{m n_{2}}^{y^{2}}}{c_{m n_{2}}^{x-y^{2}}} \\
& \cdot \sum_{i=1}^{M} \sum_{k=1}^{M} \sum_{j=1}^{N 1} \sum_{l=1}^{N 1} \sum_{h=1}^{N 2} V_{i j k l} D_{i j h} D_{k l h} .
\end{aligned}
$$

$g_{m n_{1} n_{2}}(t)$ include the linear part $g_{0 m n_{1} n_{2}}(t)$ and 1st-order perturbation parameter $\left(\varepsilon_{m n_{1} n_{2}}^{x} /\left(1+b_{m n_{1}}^{y^{1}}+b_{m n_{2}}^{y^{2}}\right)\right)^{1}$ term $g_{1 m n_{1} n_{2}}(t)$

$$
g_{m n_{1} n_{2}}(t)=g_{0 m n_{1} n_{2}}(t)+\frac{\varepsilon_{m n_{1} n_{2}}^{x}}{1+b_{m n_{1}}^{y^{1}}+b_{m n_{2}}^{y^{2}}} g_{1 m n_{1} n_{2}}(t)
$$

and lastly, $f_{m n_{1} n_{2}}(t)$ in (29) can be determined as

$$
\begin{aligned}
f_{m n_{1} n_{2}}(t)= & \cos \omega_{m n_{1} n_{2}} t \\
& +\frac{\varepsilon_{m n_{1} n_{2}}^{x}}{1+b_{n_{1} m}^{y}+b_{n_{1} n_{2}}^{z}} \frac{1}{D_{m n_{1} n_{2}}} g_{1 m n_{1} n_{2}}(t) .
\end{aligned}
$$

Thus, the circular frequency of the tri-cross string can be obtained as

$$
\begin{aligned}
& \left(\omega_{m n_{1} n_{2}}\right)^{2}=\frac{\left(\omega_{0 m}\right)^{2}+b_{m n_{1}}^{y^{1}}\left(\omega_{0 n_{1}}^{\prime}\right)^{2}+b_{m n_{2}}^{y^{2}}\left(\omega_{0 n_{2}}^{\prime \prime}\right)^{2}}{1+b_{m n_{1}}^{y^{1}}+b_{m n_{2}}^{y^{2}}} \\
& +\frac{\varepsilon_{m n_{1} n_{2}}^{x}}{1+b_{m n_{1}}^{y^{1}}+b_{m n_{2}}^{y^{2}}} \alpha_{1} \\
& =\frac{\left(\omega_{0 m}\right)^{2}+b_{m n_{1}}^{y^{1}}\left(\omega_{0 n_{1}}^{\prime}\right)^{2}+b_{m n_{2}}^{y^{2}}\left(\omega_{0 n_{2}}^{\prime \prime}\right)^{2}}{1+b_{m n_{1}}^{y^{1}}+b_{m n_{2}}^{y^{2}}} \\
& +\frac{1+\varepsilon_{m n_{1} n_{2}}^{x}}{1+b_{m n_{1}}^{y^{1}}+b_{m n_{2}}^{y^{2}}}\left(\frac{1}{4} \sum_{i=1}^{M} \sum_{j=1}^{N 1} \sum_{k=1}^{N 1} \sum_{l=1}^{N 2} \sum_{h=1}^{N 2} p_{j k l h} D_{i j l} D_{i k h}\right. \\
& +\frac{1}{4} \frac{b_{m n_{1}}^{y^{1}}}{c_{m n_{1}}^{x-y^{1}} \sum_{i=1}^{M} \sum_{k=1}^{M} \sum_{j=1}^{N 1} \sum_{l=1}^{N 2} \sum_{h=1}^{N 2} q_{i k l h} D_{i j l} D_{k j h}} \\
& \left.+\frac{1}{4} \frac{b_{m n_{2}}^{y^{2}}}{c_{m n_{2}}^{x-y^{2}}} \sum_{i=1}^{M} \sum_{k=1}^{M} \sum_{j=1}^{N 1} \sum_{l=1}^{N 1} \sum_{h=1}^{N 2} V_{i j k l} D_{i j h} D_{k l h}\right) .
\end{aligned}
$$

Therefore, the frequency of the tri-cross string is in the following form:

$$
\begin{aligned}
& f_{m n_{1} n_{2}}^{\mathrm{st}}=\frac{1}{2 \pi} \\
& \cdot \sqrt{\frac{\left(\omega_{0 m}\right)^{2}+b_{m n_{1}}^{y^{1}}\left(\omega_{0 n_{1}}^{\prime}\right)^{2}+b_{m n_{2}}^{y^{2}}\left(\omega_{0 n_{2}}^{\prime \prime}\right)^{2}}{1+b_{m n_{1}}^{y^{1}}+b_{m n_{2}}^{y^{2}}}+\frac{\varepsilon_{m n_{1} n_{2}}^{x}}{1+b_{m n_{1}}^{y^{1}}+b_{m n_{2}}^{y^{2}}} \alpha_{1}} .
\end{aligned}
$$

According to the initial conditions, the amplitude $a_{m n_{1} n_{2}}$ can be obtained by the following relation:

$$
\begin{gathered}
\sum_{m=1}^{M} \sum_{n_{1}=1}^{N 1} \sum_{n_{2}=1}^{N 2} a_{m n_{1} n_{2}} f_{m n_{1} n_{2}}(t) \sin \frac{m \pi x_{i}}{l_{0}} \sin \frac{n_{1} \pi y_{j}^{1}}{l_{1}} \\
\cdot \sin \frac{n_{2} \pi y_{k}^{2}}{l_{2}}=w_{\mathrm{st}}\left(x_{i}, y_{j}^{1}, y_{k}^{2}, 0\right),
\end{gathered}
$$

where $m=1,2, \ldots, M ; n_{1}=1,2, \ldots, N 1 ; n_{2}=1,2, \ldots$, N2.

In this study, approximate semi-analytic formulas on the natural analysis of the structural displacements and frequencies are presented. To approach the exact solution as close as possible, large value of $M, N 1, N 2$ should be chosen, where the number of the chosen sampling points on the three strings should be identical as well. To improve the accuracy of the solution derived from the above perturbation method, the order of magnitude of the parameter $\left(\varepsilon_{m n_{1} n_{2}}^{x} /\left(1+b_{m n_{1}}^{y^{1}}+b_{m n_{2}}^{y^{2}}\right)\right)$ also needs to be increased. Next we will demonstrate it with various numerical examples.

\section{Results and Discussion}

We choose the geometrical and mechanical properties of the tri-cross string as follows: $l_{0}=1 \mathrm{~m}, l_{1}=0.95 \mathrm{~m}, l_{2}=1.05 \mathrm{~m}$, $x_{1 \text {,joint }}=0.32 l_{0}, x_{2, \text { joint }}=0.64 l_{0}, y_{\text {joint }}^{1}=0.33 l_{1}, y_{\text {joint }}^{2}=0.63 l_{2}$, $E=210 \times 10^{9} \mathrm{~Pa}, \rho=0.1543 \mathrm{~kg} / \mathrm{m}, \varepsilon=0.2 \times 10^{-3}$. The three strings all have the same diameter, that is, $0.005 \mathrm{~m}$. The magnitude of the maximum displacement is taken to be $10^{-3} \mathrm{~m}$. The perturbation parameters $\left(\varepsilon_{m n_{1} n_{2}}^{x} /\left(1+b_{m n_{1}}^{y^{1}}+\right.\right.$ $\left.\left.b_{m n_{2}}^{y^{2}}\right)\right)\left(m, n_{1}, n_{2}=1,2, \ldots, N\right)$ are chosen to be much smaller than unity with a maximum value of $10^{-7}$.

In order to discuss the accuracy of the solution equation (55), Table 1 presents the first three-order natural frequencies with different $M, N 1$, and N2. It is shown that (55) with 4 or 5 perturbation terms already provides sufficient accuracy for the current tri-cross string structure. This fact confirms that the perturbation procedure with the above parameters is sufficient for the problem considered here. In the following discussions, we will choose $M=N 1=N 2=5$.

Until now, the nonlinear free vibration analysis of a tricross string with geometrical linearity cannot be accurately simulated with a commercial finite element code to provide a numerical solution because the nonlinearity from varying arc length and the coupled characteristics caused by jogged joints are quite complicated. In this work, we focus on the discussion of the nonlinearity and couple characteristic of the prestressed tri-cross string.

With similar process, we can obtain the nonlinear frequency of one single string. The frequencies of the three strings corresponding to the tri-cross string are given below in an explicit form: 
TABLE 1: The first three frequencies with different amplitudes (Hz).

\begin{tabular}{lcccccccccc}
\hline \multirow{2}{*}{$w_{\text {st }}\left(x=0.5, y_{\text {joint }}^{1}=0.33, y_{\text {joint }}^{2}=0.63,0\right) / \mathrm{m}$} & \multicolumn{3}{c}{3 terms $(N=3)$} & \multicolumn{3}{c}{4 terms $(N=4)$} & \multicolumn{3}{c}{5 terms $(N=5)$} \\
& $f_{111}^{\text {st }}$ & $f_{222}^{\text {st }}$ & $f_{333}^{\text {st }}$ & $f_{111}^{\text {st }}$ & $f_{222}^{\text {st }}$ & $f_{333}^{\text {st }}$ & $f_{111}^{\text {st }}$ & $f_{222}^{\text {st }}$ & $f_{333}^{\text {st }}$ \\
\hline $1.2 \times 10^{-3}$ & 37.74 & 75.60 & 114.53 & 37.03 & 74.03 & 111.96 & 36.95 & 73.82 & 111.63 \\
$2.4 \times 10^{-3}$ & 37.89 & 75.92 & 115.05 & 37.22 & 74.38 & 112.52 & 37.46 & 74.77 & 112.92 \\
$3.0 \times 10^{-3}$ & 38.02 & 76.16 & 115.43 & 37.56 & 74.66 & 113.95 & 37.82 & 75.47 & 114.24 \\
$4.0 \times 10^{-3}$ & 38.27 & 76.67 & 116.26 & 38.66 & 75.25 & 114.86 & 38.59 & 76.97 & 115.92 \\
\hline
\end{tabular}

$$
\begin{aligned}
& f_{m}^{\mathrm{I}}=\frac{1}{2 \pi} \sqrt{\omega_{0 m}^{2}+\frac{a_{m}^{2}\left(E A+T_{0}\right) l_{0}^{-2}}{2 \rho T_{0}^{2}}\left(\frac{m \pi}{l_{0}}\right)^{2} \cdot\left[\frac{1}{2} a_{m}^{2} y_{1}^{m}\left(\frac{m \pi}{l_{0}}\right)^{2}+\sum_{i=1}^{N} \frac{1}{2} a_{i}^{2} y_{1}^{i}\left(\frac{i \pi}{l_{0}}\right)^{2}\right]}, \\
& f_{m}^{\mathrm{II}}=\frac{1}{2 \pi} \sqrt{\left(\omega_{0 m}^{\prime}\right)^{2}+\frac{a_{m}^{2}\left(E A_{1}+T_{1}\right) l_{0}^{\prime-2}}{2 \rho T_{1}^{2}}\left(\frac{m \pi}{l_{1}}\right)^{2} \cdot\left[\frac{1}{2} a_{m}^{2} x_{1}^{m}\left(\frac{m \pi}{l_{1}}\right)^{2}+\sum_{i=1}^{N} \frac{1}{2} a_{i}^{2} x_{1}^{i}\left(\frac{i \pi}{l_{1}}\right)^{2}\right]}, \\
& f_{m}^{\mathrm{III}}=\frac{1}{2 \pi} \sqrt{\left(\omega_{0 m}^{\prime \prime}\right)^{2}+\frac{a_{m}^{2}\left(E A_{2}+T_{2}\right) l_{0}^{\prime-2}}{2 \rho\left(T_{2}\right)^{2}}\left(\frac{m \pi}{l_{2}}\right)^{2} \cdot\left[\frac{1}{2} a_{m}^{2} y_{2}^{m}\left(\frac{m \pi}{l_{2}}\right)^{2}+\sum_{i=1}^{N} \frac{1}{2} a_{i}^{2} y_{2}^{i}\left(\frac{i \pi}{l_{2}}\right)^{2}\right]} .
\end{aligned}
$$

In the following discussions, the natural frequencies of the tri-cross string will be compared with those of the corresponding constituent single strings. Comparing (55) with (57), it can be seen that (55) includes not only linear frequency $\left[\left(\omega_{0 m}\right)^{2}+b_{m n_{1}}^{y^{1}}\left(\omega_{0 n_{1}}^{\prime}\right)^{2}+b_{m n_{2}}^{y^{2}}\left(\omega_{0 n_{2}}^{\prime \prime}\right)^{2}\right] /\left(1+b_{m n_{1}}^{y^{1}}+b_{m n_{2}}^{y^{2}}\right)$, combined with the linear frequencies from the three single strings, but also nonlinear fluctuation $\left(\left(\varepsilon_{m n_{1} n_{2}}^{x} /\left(1+b_{m n_{1}}^{y^{1}}+\right.\right.\right.$ $\left.\left.\left.b_{m n_{2}}^{y^{2}}\right)\right) \alpha_{1}\right)$, where the terms such as $p_{j k l h} D_{i j l} D_{i k h}$ reflect the coupling characteristics from the jogged strings.

In the following sections, we will discuss the nonlinear frequencies of the tri-cross string by varying structural parameters, such as the vibration amplitude, the diameter of one string, the diameters of two strings, the length of one string, and the lengths of two strings.

Figure 4 shows the first four natural frequencies of the whole tri-cross string $\left(f_{111}^{\text {st }}, f_{222}^{\text {st }}, f_{333}^{\text {st }}, f_{444}^{\text {st }}\right)$ with different initial amplitudes $w_{\text {st }}\left(x=0.5 l_{0}, y_{\text {joint }}^{1}, y_{\text {joint }}^{2}, 0\right)$. First, the natural frequencies with the same mode in the three constituent strings, that is, $f_{111}^{\text {st }}, f_{222}^{\text {st }}, f_{333}^{\text {st }}, f_{444}^{\text {st }}$, are chosen in the discussion. It is seen that the natural frequencies of the tri-cross string and the three single strings increase with increasing amplitudes. Moreover, the frequencies of the tri-cross string are among those of the three single strings, reflecting the averaging effect of the three strings. It is one kind of the coupling effect of the tri-cross string. The total frequencies are not only the average of the nonlinear frequencies of the three constituent single strings in any way. To some extent, the total frequency $f_{m n_{1} n_{2}}^{\text {st }}$ can be regarded as one kind of nonlinear combination of frequencies of the three constituent single strings. Therefore, the variation tendency of the frequency of the tri-cross string presents a nonlinear response by varying the amplitudes of the tri-cross string shown in Figure 4. If geometries of the constituent single strings are changed, the differences among the tri-cross string and the three single strings become rather complicated according to the results from our calculations.

Figure 5 shows the influence of increasing the diameter of one constituent string on the first four natural frequencies of the strings, with the initial displacement of $1 \times 10^{-3} \mathrm{~m}$ at the point with the maximum magnitude. By setting $l_{0}=1 \mathrm{~m}$, $l_{1}=0.95 \mathrm{~m}, l_{2}=1.05 \mathrm{~m}$, the initial natural frequencies of the single strings are different from each other, which has been shown in Figure 5. In the case of increasing the diameter of one single string, its own frequencies increase and the frequencies of the whole tri-cross string also increase. It is shown that the change of the frequencies of the whole tri-cross string accompanies the varying of diameter of $y^{1}$ string. In contrast, the $x$-string and $y^{2}$-string have not been changed while changing the diameter of $y^{1}$-string. The results demonstrate that changing the diameter of one single string does not change the frequencies of the whole structure and the frequencies of the other two constituent strings too much.

In the following, we study the effect of changing the diameters of two strings. Figure 6 depicts the profiles of the first four natural frequencies of the strings, by increasing the diameters of two constituent strings (with $d_{2}=d_{3}\left(A_{1}=\right.$ $\left.\left.(1 / 4) \pi d_{2}^{2}, A_{2}=(1 / 4) \pi d_{3}^{2}\right)\right)$, with the initial displacement of $1 \times 10^{-3} \mathrm{~m}$ at the point in maximum magnitude. By setting $l_{0}=$ $1 \mathrm{~m}, l_{1}=0.95 \mathrm{~m}, l_{2}=1.05 \mathrm{~m}$, the initial natural frequencies of the single strings are different from each other, which 

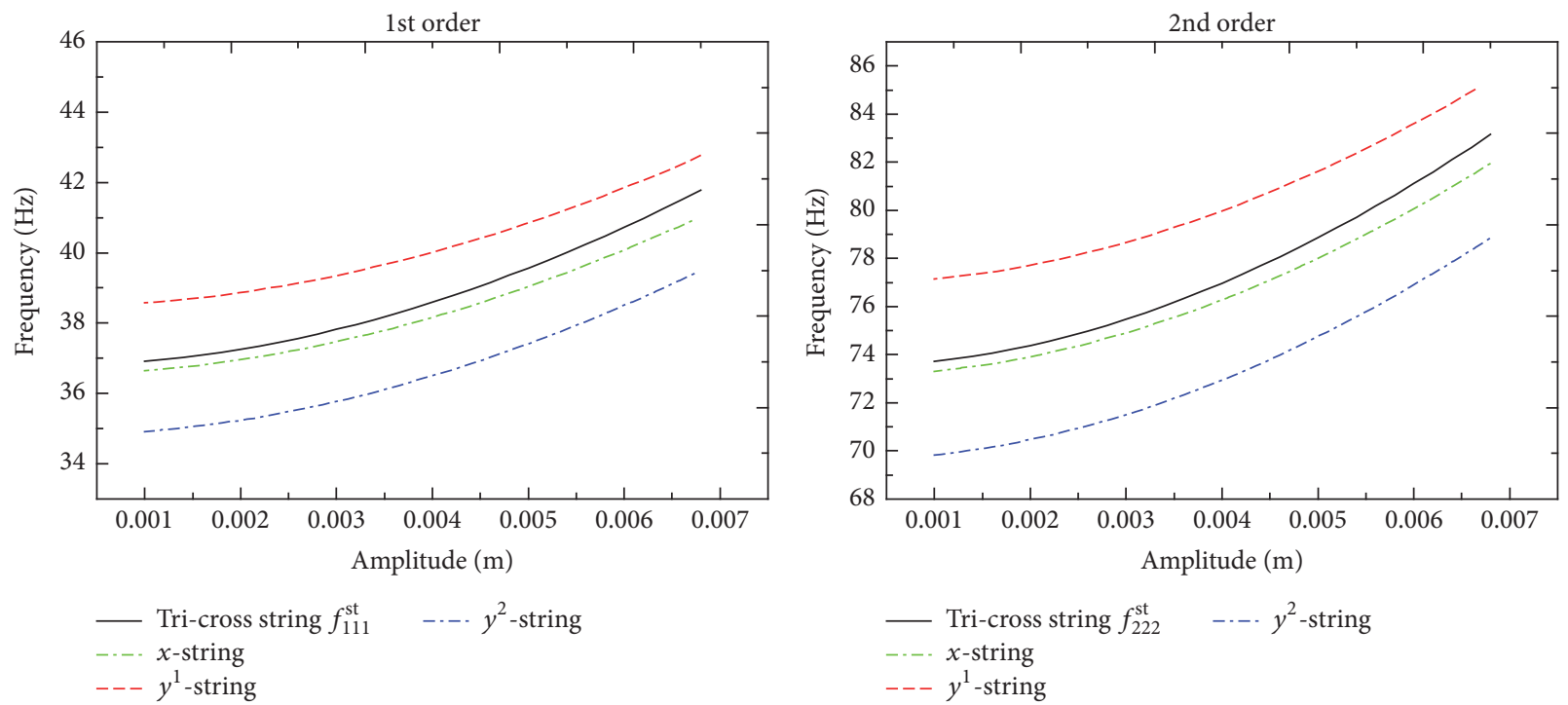

(a)

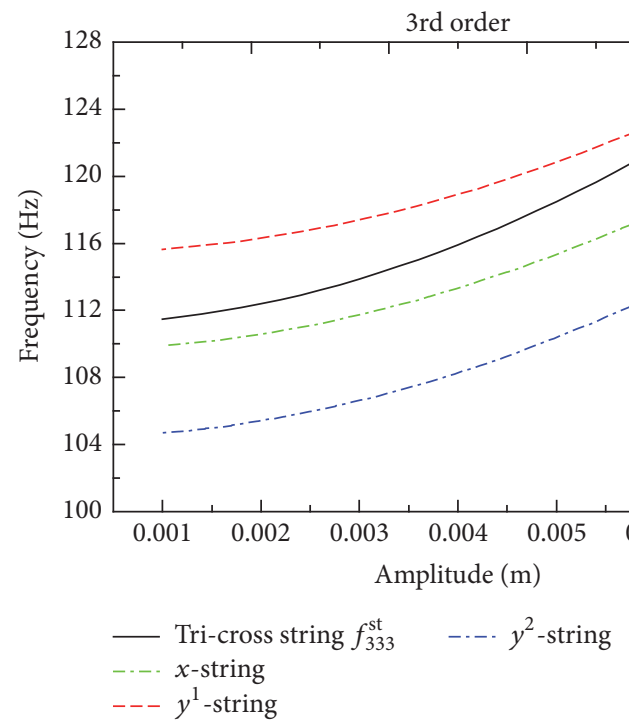

(c)

(b)

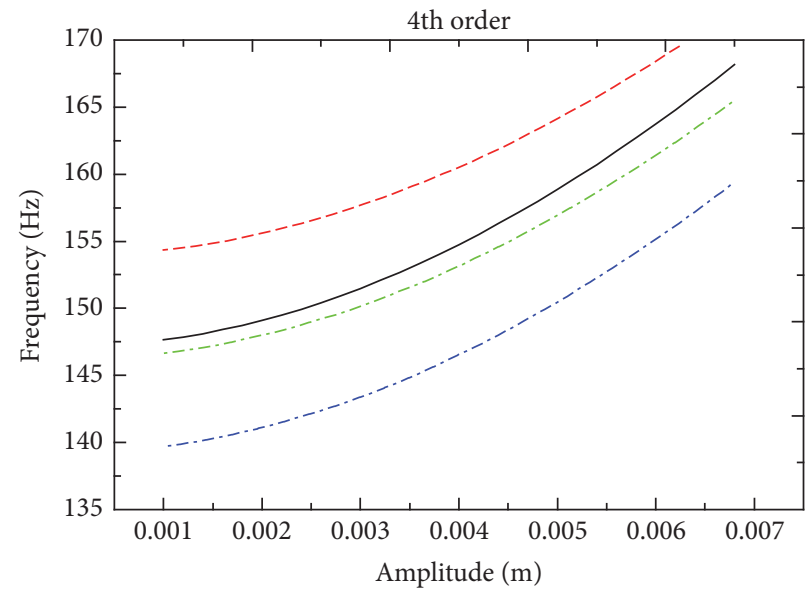

Tri-cross string $f_{444}^{\text {st }} \quad \ldots-\cdot y^{2}$-string
$---x$-string
$---y^{1}$-string

(d)

FIgURE 4: The influence of the vibration amplitude $w_{\text {st }}\left(x=0.5 l_{0}, y_{\text {joint }}^{1}, y_{\text {joint }}^{2}, 0\right)$ on the first four frequencies of the strings: (a) the first mode $f_{111}^{\text {st }}$, (b) the second mode $f_{222}^{\text {st }}$, (c) the third mode $f_{333}^{\text {st }}$, and (d) the fourth mode $f_{444}^{\text {st }}$.

has been shown in Figure 6. When the diameters of these two strings increase, their own frequencies increase and the frequencies of the whole tri-cross string also increase. Similar to the case of changing the diameter of only one string, the tri-cross string changes its frequencies less than that of the $y^{1}$-string. The frequency of the $y^{2}$-string does not change too much as well. This confirms the important fact again that the frequencies of tri-cross string cannot be treated as a mere combination of the nonlinear frequencies of the three constituent strings in any way.
In addition to the diameters, the lengths of the strings also have profound influence on the vibration of the tri-cross string; see Figure 7 for the effect of the length ratio of the two strings $l_{1} / l_{0}$ (here $l_{0}$ and $l_{2}$ keep constant) on the first four natural frequencies of the cross string $\left(f_{111}^{\mathrm{st}}, f_{222}^{\mathrm{st}}, f_{333}^{\mathrm{st}}, f_{444}^{\mathrm{st}}\right)$, with the initial displacement being $1 \times 10^{-3} \mathrm{~m}$ at the point with the maximum magnitude. With the consideration of increasing the string length ratio, it is indicated that the natural frequencies of the tri-cross string at different modes will decrease. The length ratio factor by varying the length 


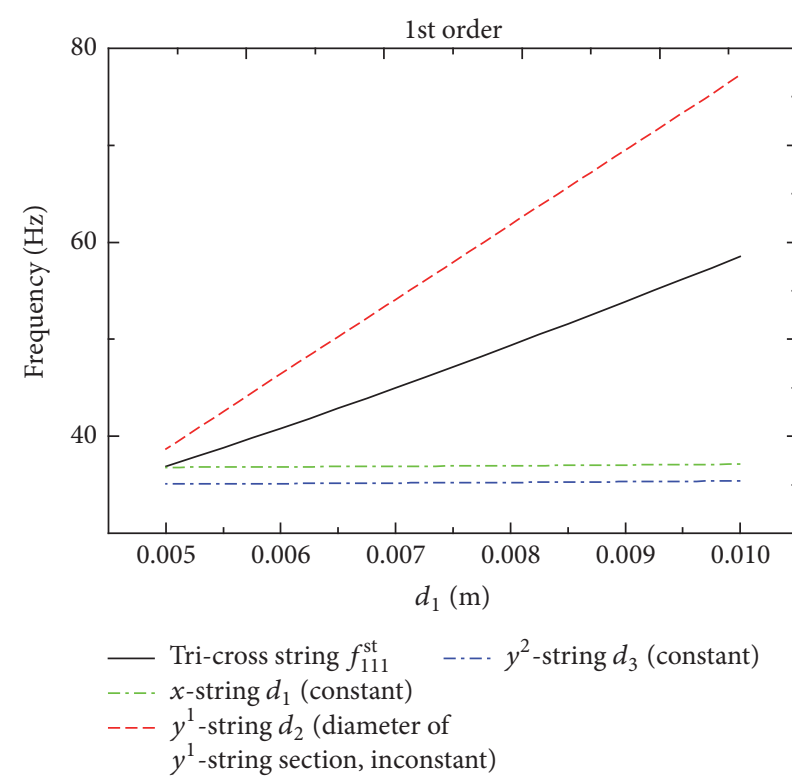

(a)

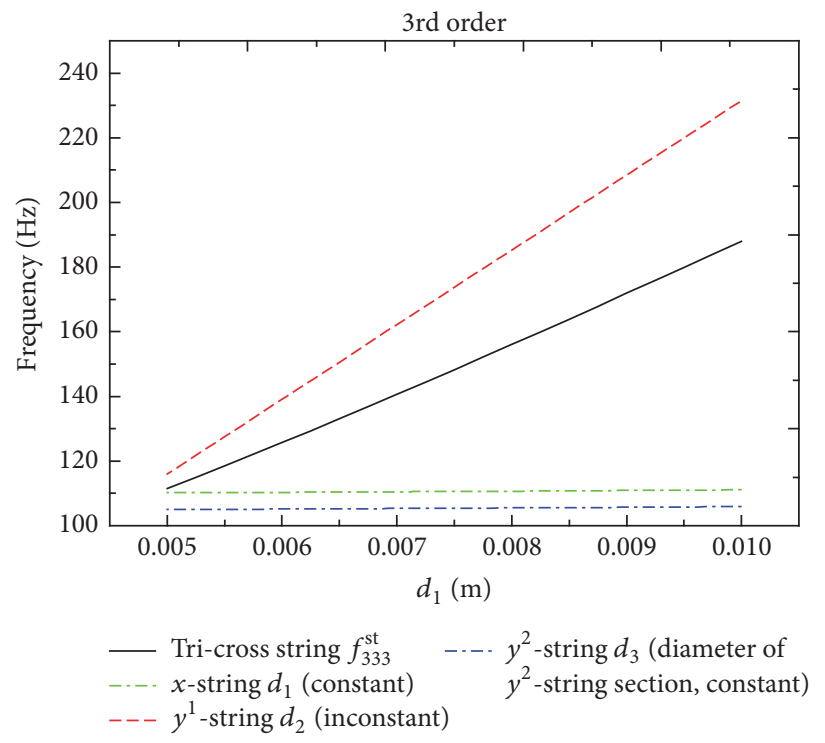

(c)

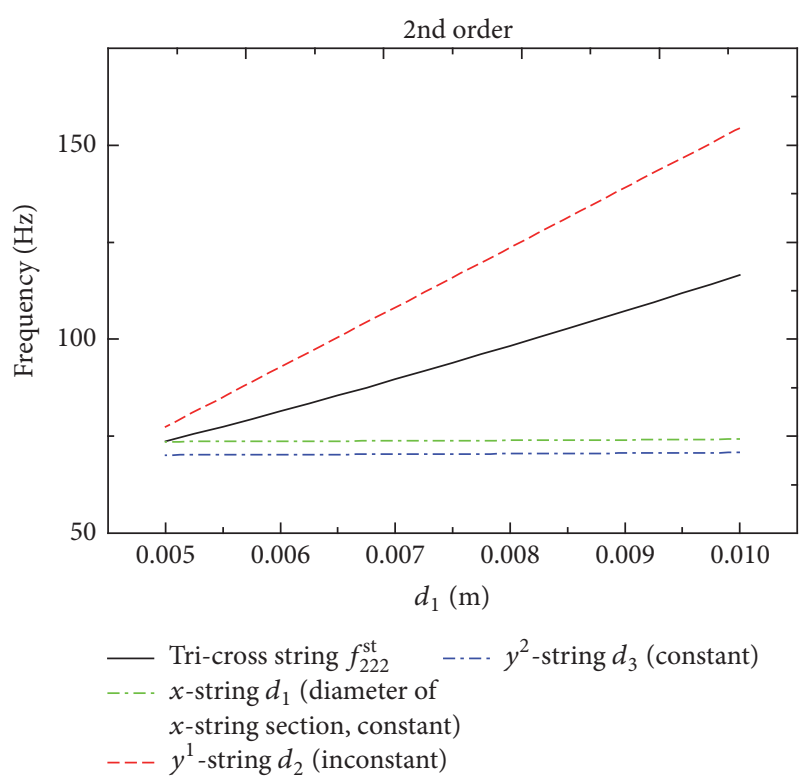

(b)

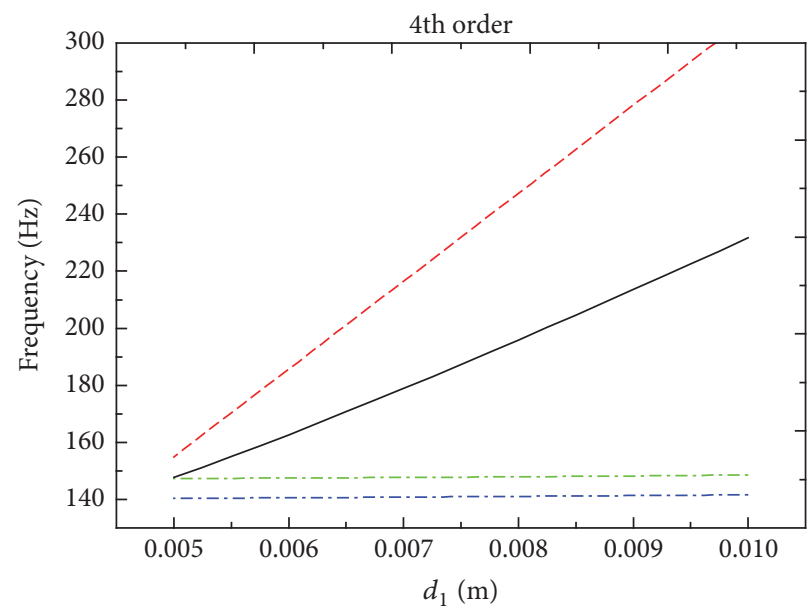

- Tri-cross string $f_{444}^{\mathrm{st}} \quad \ldots-\cdot y^{2}$-string $d_{3}$ (constant)
$-\ldots$-string $d_{1}$ (constant)
$---y^{1}$-string $d_{2}$ (inconstant)

(d)

Figure 5: The influence of the diameter of one constituent string on the first four frequencies of the strings: (a) the first mode $f_{111}^{\text {st }}$, (b) the second mode $f_{222}^{\text {st }}$, (c) the third mode $f_{333}^{\text {st }}$, and (d) the fourth mode $f_{444}^{\text {st }}$.

of one string provides pronounced effect on the tri-cross string, however, not as that on the varying single string. That is to say, the frequency of the tri-cross string is determined by the properties of its constituent strings, but the response sensitivity of the tri-cross string on local structural variation decreases due to its coupling characteristics and nonlinear response.

Furthermore, we increase the lengths of $x$-string and $y^{2}$ string proportionally. The first four natural frequencies of the tri-cross string $\left(f_{111}^{\mathrm{st}}, f_{222}^{\mathrm{st}}, f_{333}^{\mathrm{st}}, f_{444}^{\mathrm{st}}\right)$ resulting from the influence of the length ratio of the two strings $l_{1} / l_{0}, l_{2} / l_{0}$ (here $l_{0}$ and $l_{2} / l_{1}$ keep constant) are shown in Figure 8 , where the initial displacement is $1 \times 10^{-3} \mathrm{~m}$ at the point with the maximum magnitude. With the consideration of increasing the string length ratio, it is indicated that the natural frequencies of the tri-cross string at different modes will decrease. Compared with the results shown in Figure 7, the frequencies of tri-cross string are much closer to the frequencies of the single strings which increase its own lengths. The reason could be that the variation of the two 


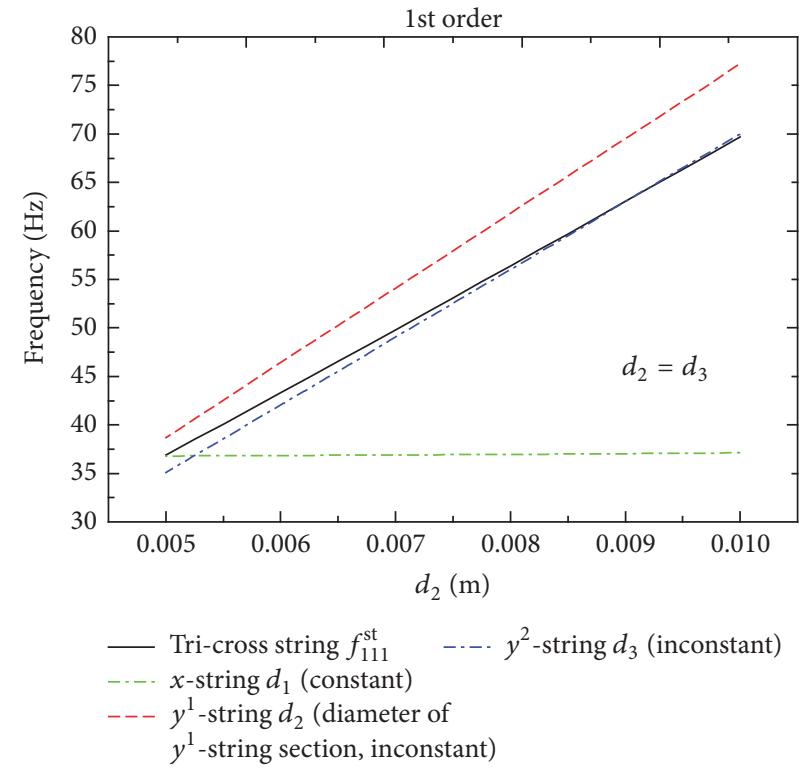

(a)

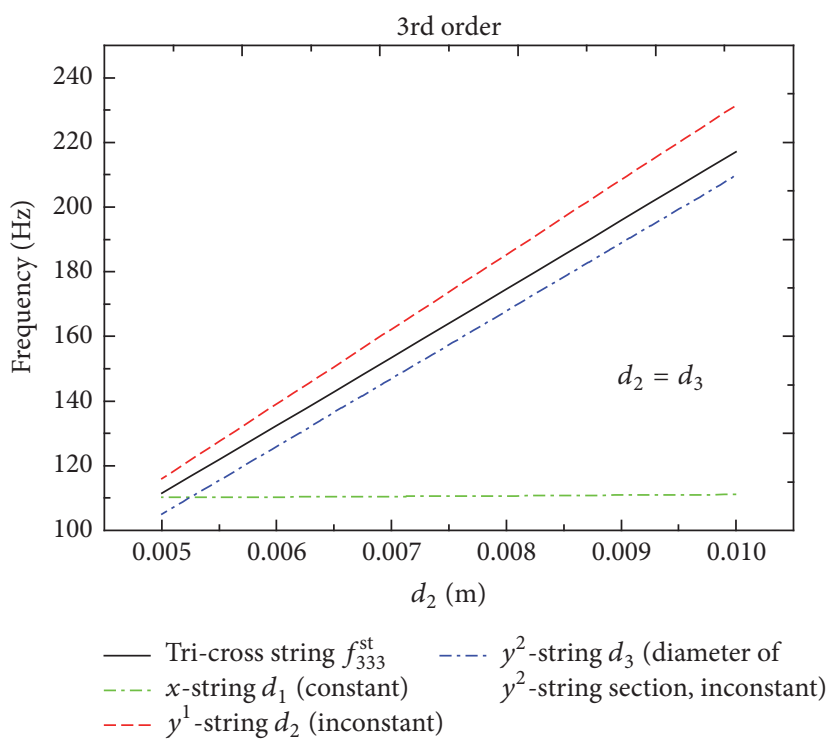

(c)

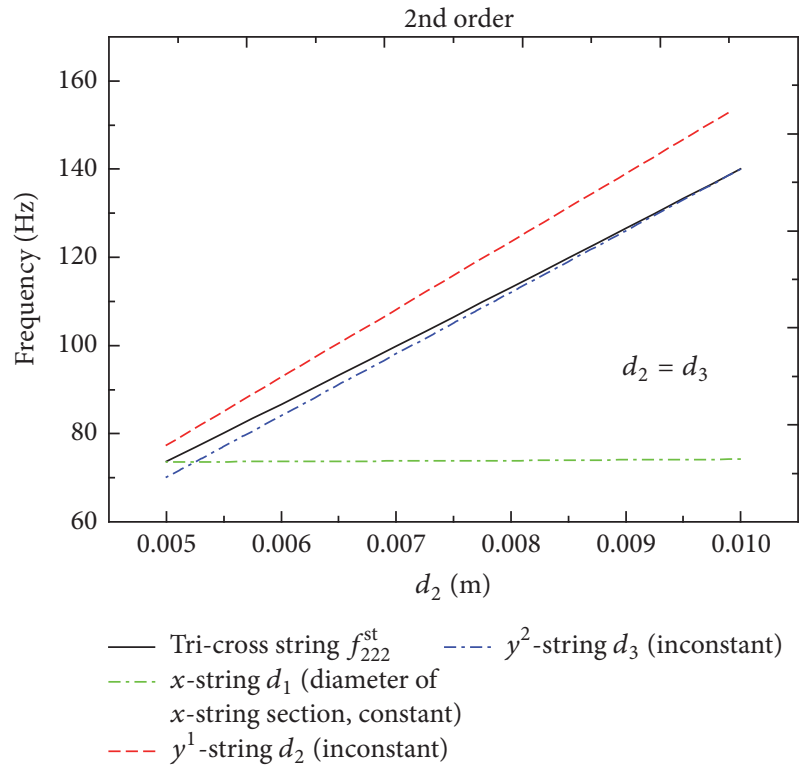

(b)

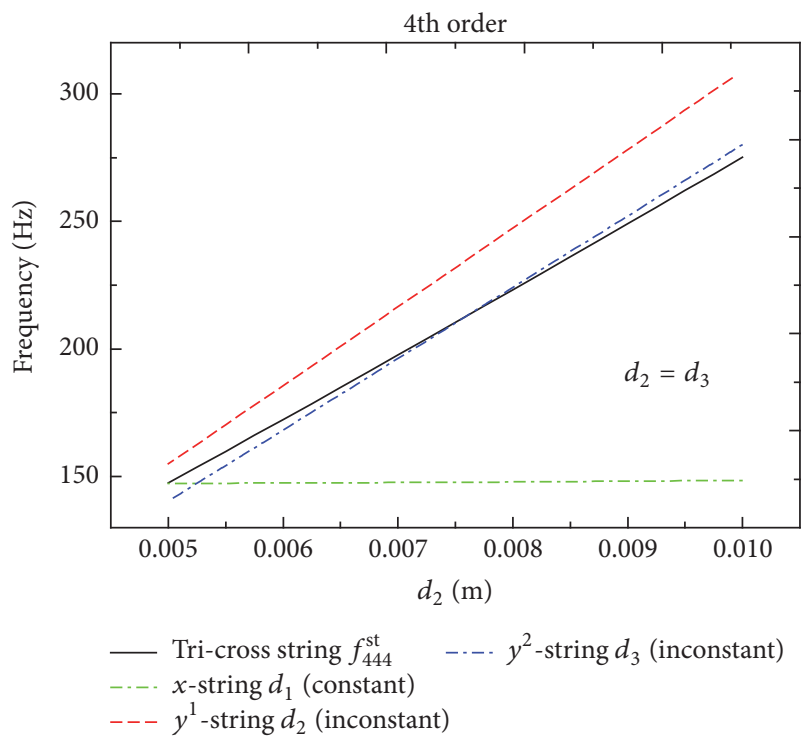

(d)

Figure 6: The influence of diameters of two constituent strings on the first four frequencies of the strings: (a) the first mode $f_{111}^{\text {st }}$, (b) the second mode $f_{222}^{\text {st }}$, (c) the third mode $f_{333}^{\text {st }}$, and (d) the fourth mode $f_{444}^{\text {st }}$.

constituent strings is more effective on the whole structure than that of single constituent string.

Figure 9 shows the first four natural frequencies of the tri-cross string $\left(f_{212}^{\text {st }}, f_{222}^{\text {st }}, f_{232}^{\text {st }}, f_{242}^{\text {st }}\right)$ with different initial amplitudes $w_{\text {st }}\left(x=0.5 l_{0}, y_{\text {joint }}^{1}, y_{\text {joint }}^{2}, 0\right)$. It is also shown that the natural frequencies of the tri-cross string and the three single strings increase with increasing amplitudes. More importantly, the frequencies $\left(f_{212}^{\mathrm{st}}, f_{222}^{\mathrm{st}}, f_{232}^{\mathrm{st}}, f_{242}^{\mathrm{st}}\right)$ show that the order of the modes is not a simple integer. It agrees with (55), where the subscript and symbol $m n_{1} n_{2}$ in the expression provide a multiple order of the natural frequencies of the tri-cross string. In another way, the subscript and symbol $m n_{1} n_{2}$ in the expression mean the different orders of modes of the constituent strings. Therefore, the results in Figure 9 and (55) reflect the coupling characteristics that are the contribution from the different orders of modes of the constituent strings. 

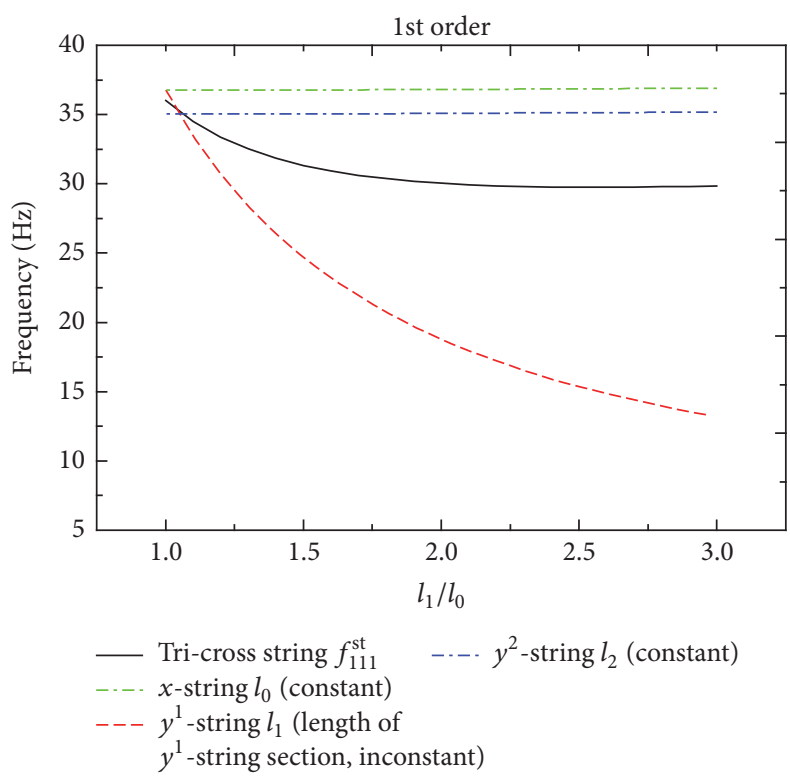

(a)

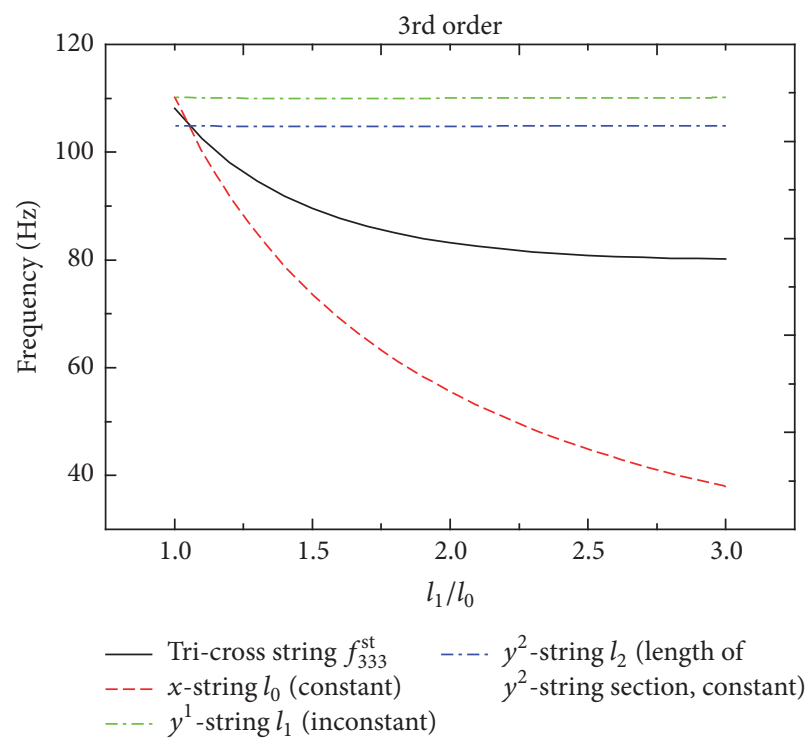

(c)

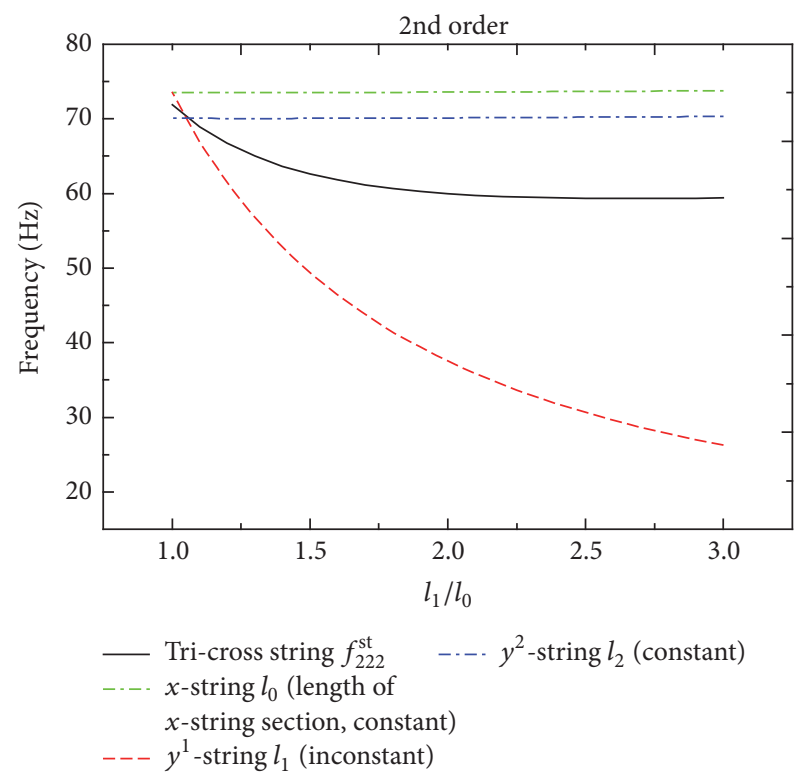

(b)

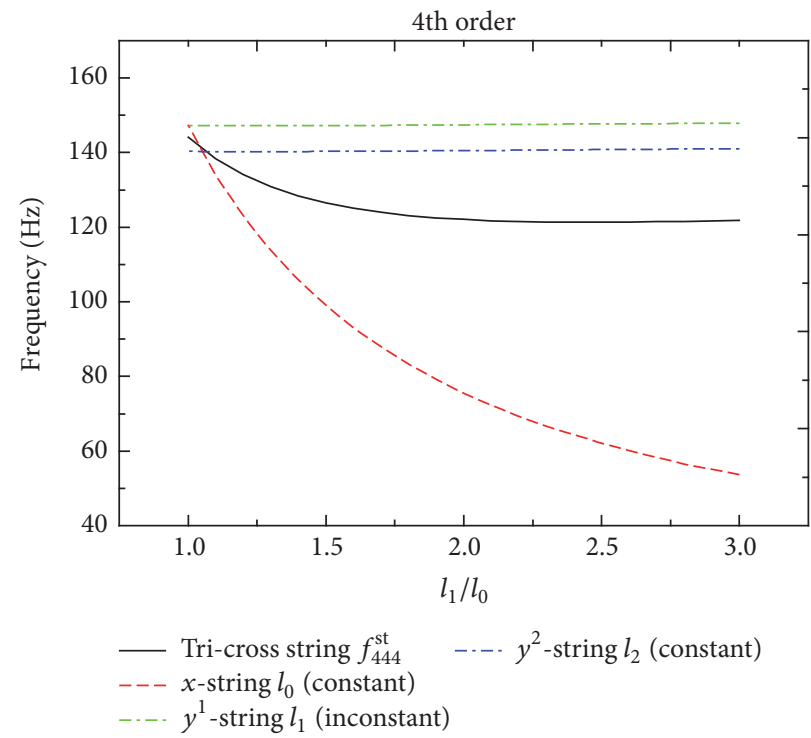

(d)

FIGURE 7: The influence of the length of one constituent string on the first four frequencies of the strings: (a) the first mode $f_{111}^{\text {st }}$, (b) the second mode $f_{222}^{\text {st }}$, (c) the third mode $f_{333}^{\text {st }}$, and (d) the fourth mode $f_{444}^{\text {st }}$.

\section{Conclusion}

In this paper, we derived the governing equations of the tricross string from Hamilton's principle. The analytical solution of the coupled structure is obtained by using the perturbation method. Furthermore, the accuracy and convergence analysis of the analytical solution has been carefully conducted. As for the properties of natural frequencies of the vibrating tricross string, the results show that the overall feature of the tri-cross string is combined with the nonlinear characteristics and coupling features; that is, the natural frequencies of the tri-cross string are composed of the linear natural frequencies of the three constituent strings and the nonlinear parts. For the nonlinear part, we use the analytical solution to study the effect of the structural parameters on the nonlinear frequencies of the tri-cross string, such as vibration amplitudes, diameters and lengths, and the different orders of natural modes of the constituent strings. The results demonstrated that the contribution of each constituent string to the tri-cross natural frequency is in different proportions. In addition, 


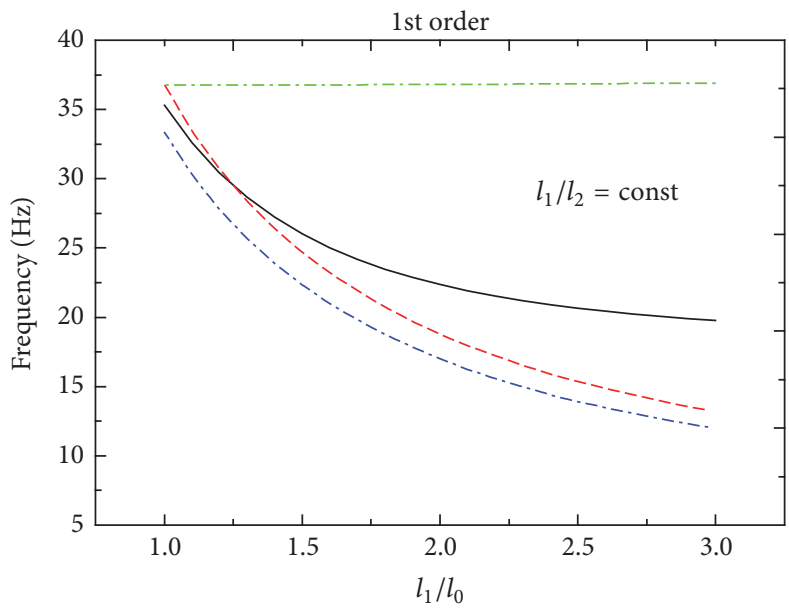

$\begin{aligned} \text { — } & \text { Tri-cross string } f_{111}^{\text {st }} \quad \ldots-\cdots y^{2} \text {-string } l_{2} \text { (inconstant) } \\ --\cdot & x \text {-string } l_{0} \text { (constant) } \\ --- & y^{1} \text {-string } l_{1} \text { (length of } \\ & y^{1} \text {-string section, inconstant) }\end{aligned}$

(a)

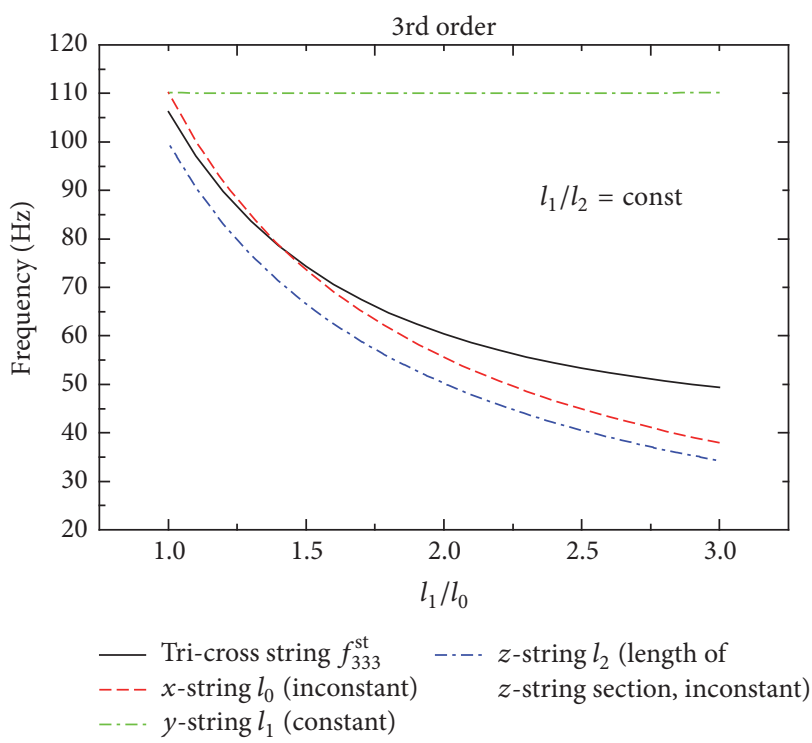

(c)

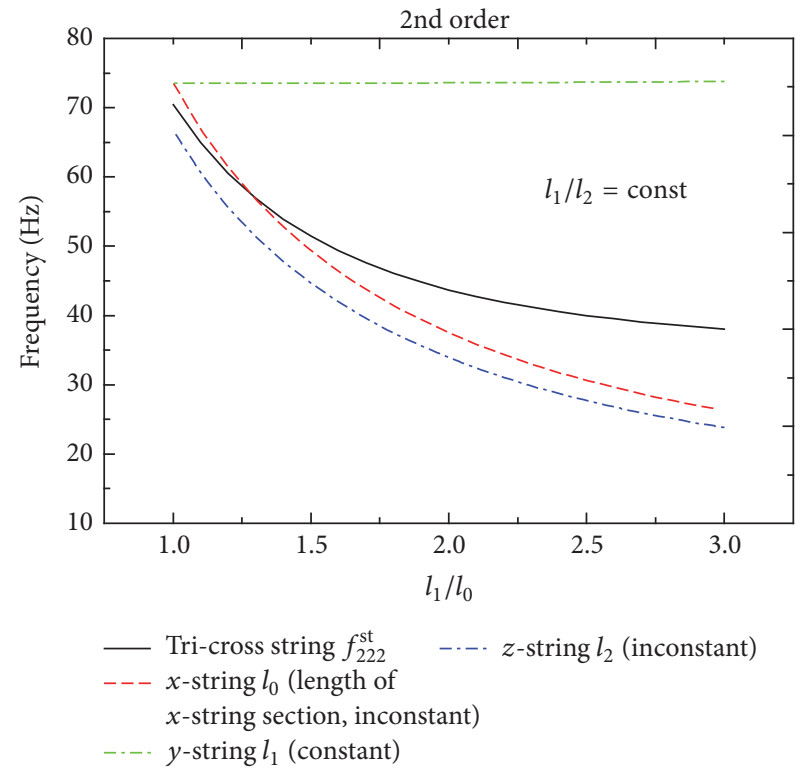

(b)

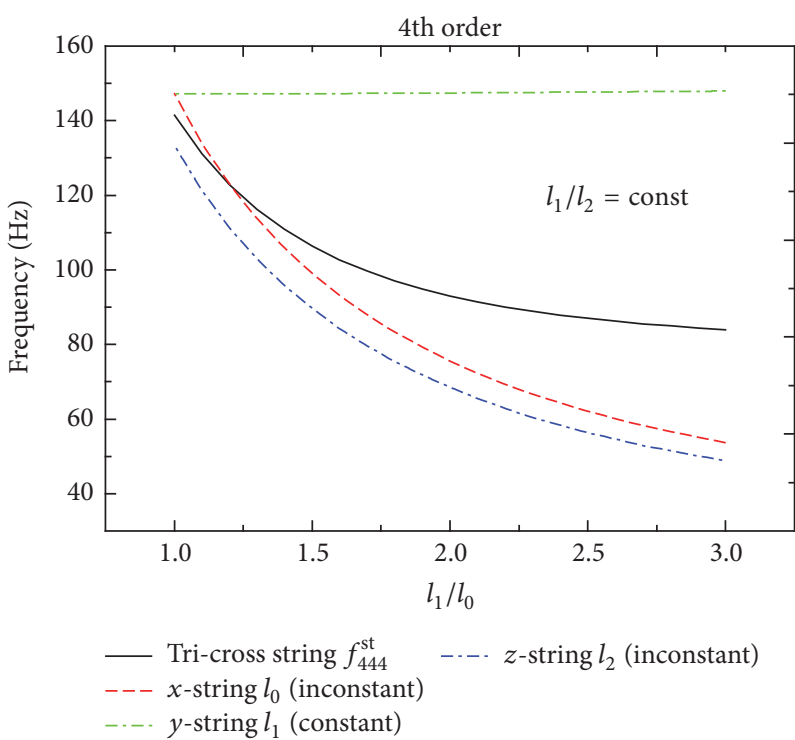

(d)

FIGURE 8: The influence of lengths of two constituent strings on the first four frequencies of the strings: (a) the first mode $f_{111}^{\text {st }}$, (b) the second mode $f_{222}^{\text {st }}$, (c) the third mode $f_{333}^{\text {st }}$, and (d) the fourth mode $f_{444}^{\text {st }}$.

the frequencies of the tri-cross string are dependent on the material properties of its constituent strings as well. More importantly, the configuration of a string net or space antennas (here the simplest net, i.e., a tri-cross string) tends to decrease the sensitivity to local structural variation. The solution obtained in this work may provide a theoretical reference for similar free vibration problem of net structures such as space antennas with flexible strings.

\section{Conflicts of Interest}

The authors declare that they have no conflicts of interest.

\section{Acknowledgments}

The authors are grateful to Professor Shibutani (Osaka University), Dr. Bo Ni (Brown University), Dr. Xin Qin 

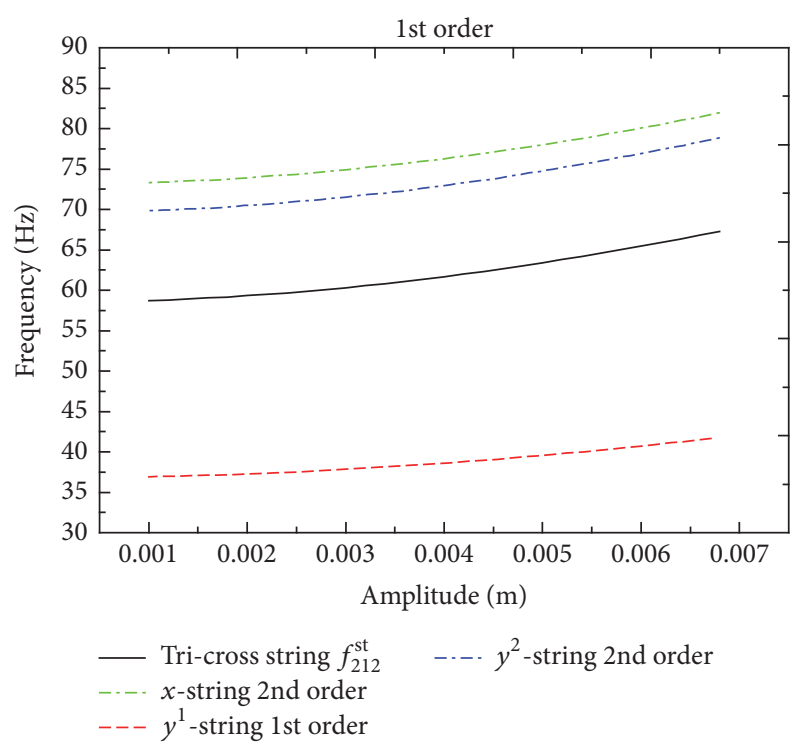

(a)

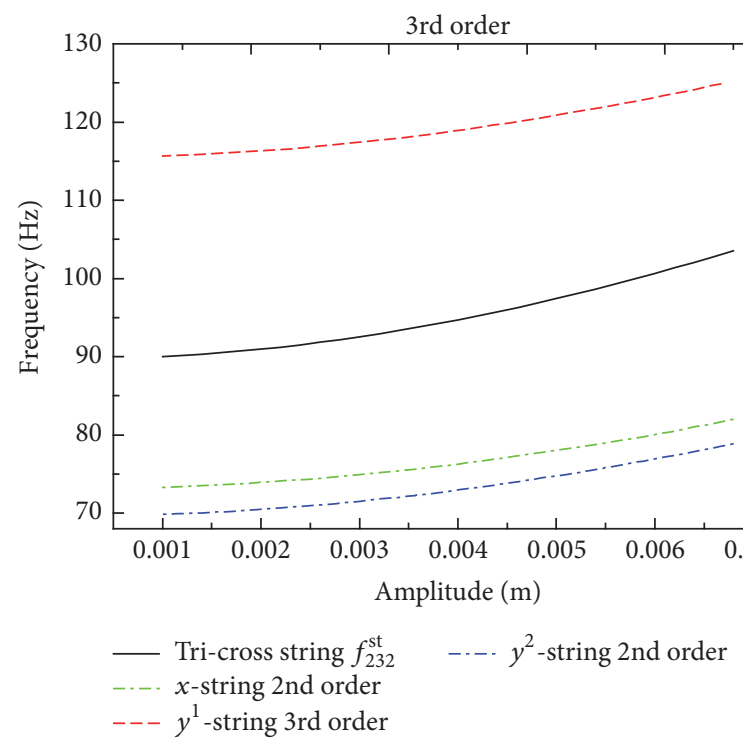

(c)

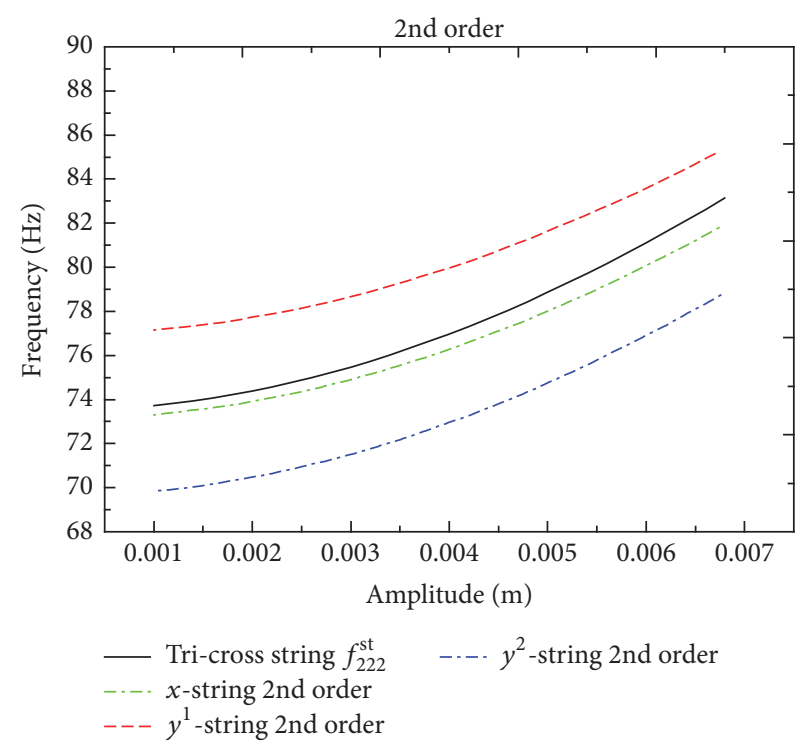

(b)

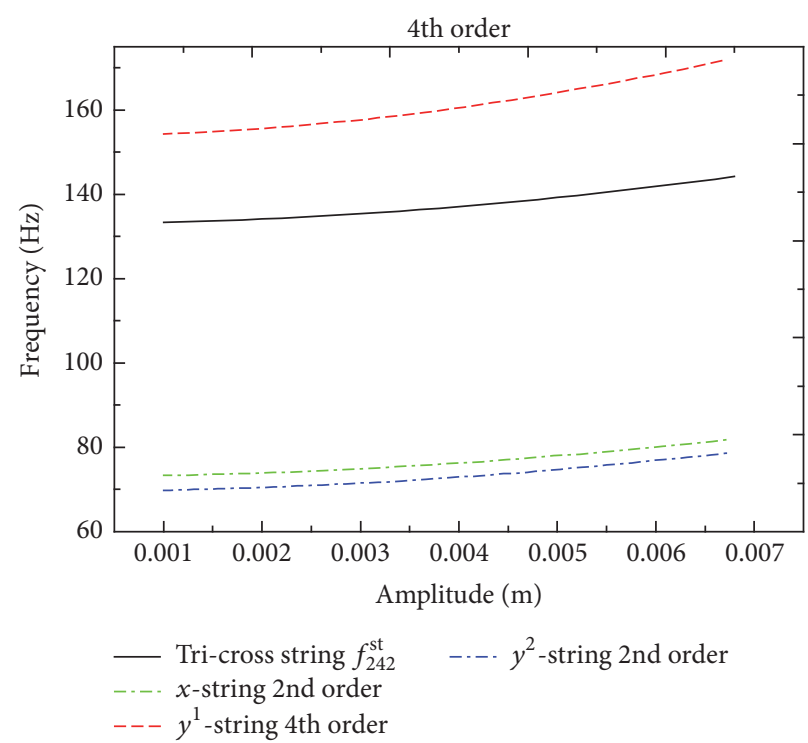

(d)

FIGURE 9: The influence of the vibration amplitude $w_{\text {st }}\left(x=0.5 l_{0}, y_{\text {joint }}^{1}, y_{\text {joint }}^{2}, 0\right)$ on the first four frequencies of the strings: (a) the first mode $f_{212}^{\text {st }}$, (b) the second mode $f_{222}^{\text {st }}$, (c) the third mode $f_{232}^{\text {st }}$, and (d) the fourth mode $f_{242}^{\text {st }}$.

(Northwestern University), Dr. Xing Li (École Polytechnique Fédérale de Lausanne), and Dr. Chao Ling (Mines ParisTech) for helpful discussion and stimulating advice on this study.

\section{References}

[1] B. Pan, J. Lu, and F. Shang, "Frequency analysis of nonlinear free vibration of a cross string," Nonlinear Dynamics, vol. 72 , no. 4 , pp. 717-728, 2013.

[2] L.-Q. Chen and H. Ding, "Two nonlinear models of a transversely vibrating string," Archive of Applied Mechanics, vol. 78, no. 5, pp. 321-328, 2008.
[3] T. C. Molteno and N. B. Tufillaro, "An experimental investigation into the dynamics of a string," American Journal of Physics, vol. 72, no. 9, pp. 1157-1169, 2004.

[4] A. W. Leissa and A. M. Saad, "Large amplitude vibrations of strings," Journal of Applied Mechanics, vol. 61, no. 2, pp. 296301, 1994.

[5] Y. Xiong and S. G. Hutton, "Vibration and Stability Analysis of a Multi-guided Rotating String," Journal of Sound and Vibration, vol. 169, no. 5, pp. 669-683, 1994.

[6] Y. M. Ram and J. Caldwell, "Free vibration of a string with moving boundary conditions by the method of distorted images," Journal of Sound and Vibration, vol. 194, no. 1, pp. 35-47, 1996. 
[7] Y. Terumichi, M. Ohtsuka, M. Yoshizawa, Y. Fukawa, and Y. Tsujioka, "Nonstationary Vibrations of a String with TimeVarying Length and a Mass-Spring System Attached at the Lower End," Nonlinear Dynamics, vol. 12, no. 1, pp. 39-55, 1997.

[8] J. Ahn, "A vibrating string with dynamic frictionless impact," Applied Numerical Mathematics, vol. 57, no. 8, pp. 861-884, 2007.

[9] S. E. Khadem and M. Rezaee, "Non-linear free vibration analysis of a string under bending moment effects using the perturbation method," Journal of Sound and Vibration, vol. 254, no. 4, pp. 677-691, 2002.

[10] G. F. Carrier, "On the non-linear vibration problem of the elastic string," Quarterly of Applied Mathematics, vol. 3, pp. 157-165, 1945.

[11] G. F. Carrier, "A note on the vibrating string," Quarterly of Applied Mathematics, vol. 7, pp. 97-101, 1949.

[12] H. Tanaka, "Design optimization studies for large-scale contoured beam deployable satellite antennas," Acta Astronautica, vol. 58, no. 9, pp. 443-451, 2006.

[13] H. Tanaka, "Surface error measurements of reconfigurable antennas based on antenna gain analyses," Space Technol. Jpn, vol. 7, pp. 19-25, 2008.

[14] C. G. Wang, Z. M. Xia, and H. F. Tan, "Initial shape design and stability analysis of rib for inflatable deployable reflector," AIAA Journal, vol. 53, no. 2, pp. 486-492, 2015.

[15] T. Cai, R. Mukherjee, and A. R. Dia, "Vibration suppression in a simple tension-aligned array structure," AIAA Journal, vol. 52, no. 3, pp. 504-515, 2014.

[16] A. H. Nayfeh and D. T. Mook, Nonlinear Oscillations, John Wiley \& Sons, New York, NY, USA, 1979.

[17] A. H. Nayfeh, Introduction to perturbation techniques, WileyInterscience, New York, NY, USA, 1981. 


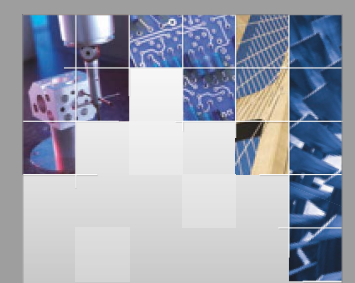

\section{Enfincering}
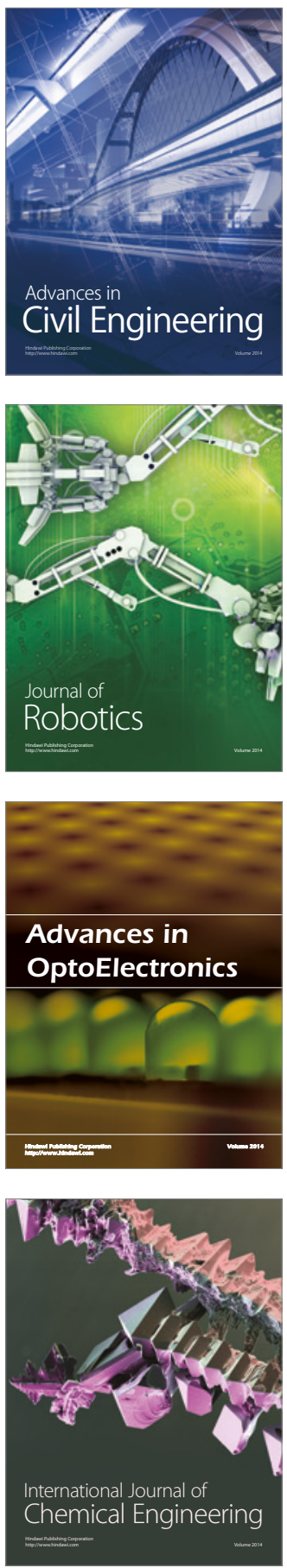

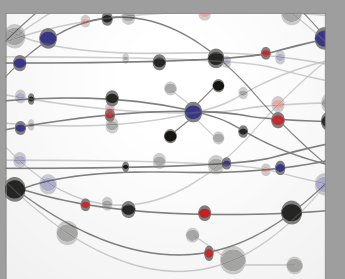

The Scientific World Journal

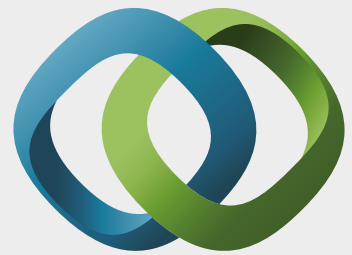

\section{Hindawi}

Submit your manuscripts at

https://www.hindawi.com
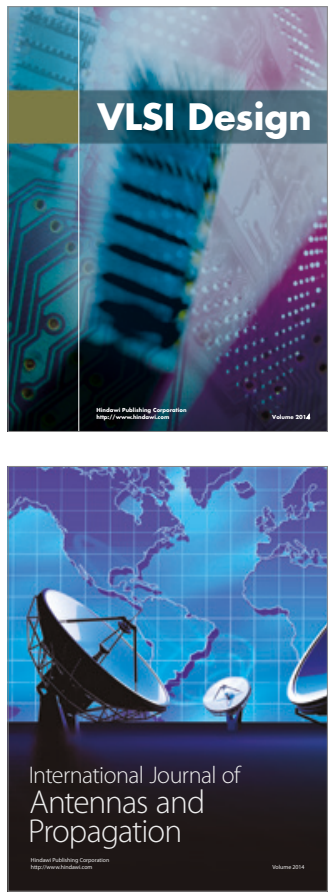

\section{Rotating}

Machinery
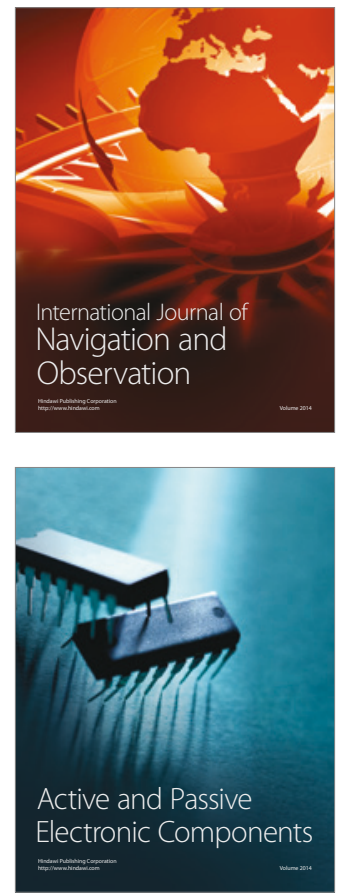
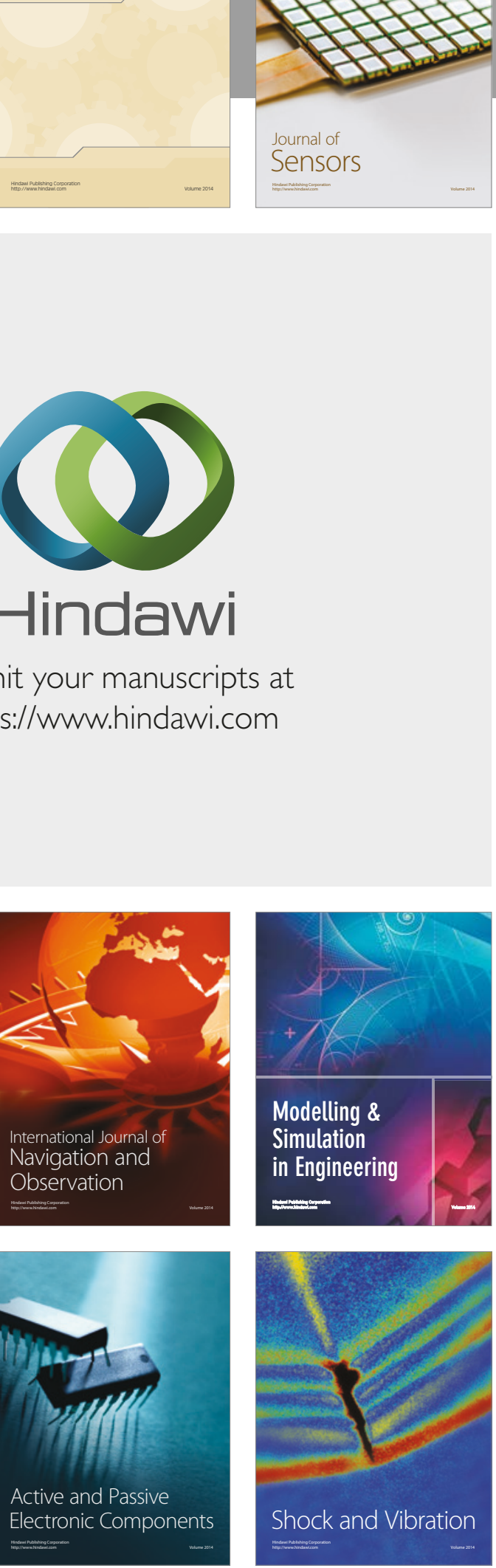
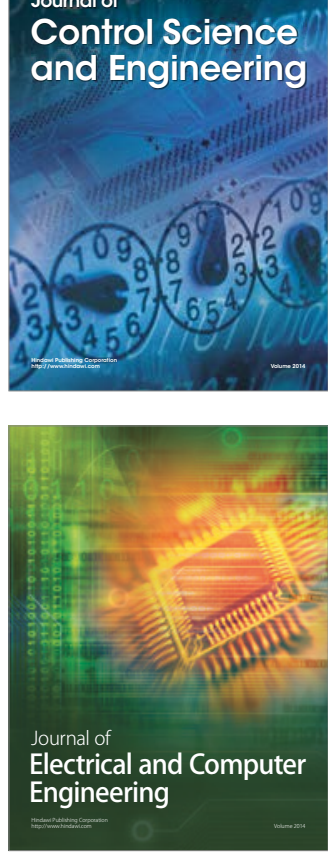

Distributed

Journal of

Control Science

and Engineering
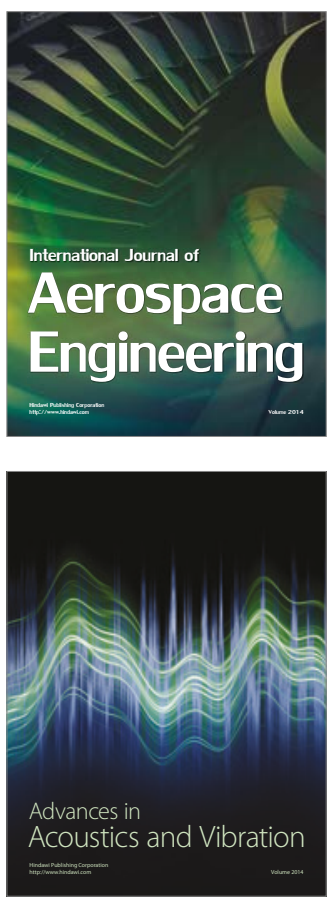

Sensor Networks 